November 2010

MIT-CTP-4193

\title{
Frame-like Geometry of Double Field Theory
}

\author{
Olaf Hohm and Seung Ki Kwak \\ Center for Theoretical Physics \\ Massachusetts Institute of Technology \\ Cambridge, MA 02139, USA \\ ohohm@mit.edu, sk_kwak@mit.edu
}

\begin{abstract}
We relate two formulations of the recently constructed double field theory to a frame-like geometrical formalism developed by Siegel. A self-contained presentation of this formalism is given, including a discussion of the constraints and its solutions, and of the resulting Riemann tensor, Ricci tensor and curvature scalar. This curvature scalar can be used to define an action, and it is shown that this action is equivalent to that of double field theory.
\end{abstract}




\section{Contents}

$\begin{array}{lll}1 & \text { Introduction } & 1\end{array}$

2 Geometrical frame formalism 4

2.1 Generalized Lie derivatives, Courant bracket and frame fields . . . . . . . . . . . . 4

$2.2 G L(D) \times G L(D)$ connections and constraints $\ldots \ldots \ldots \ldots \ldots \ldots$



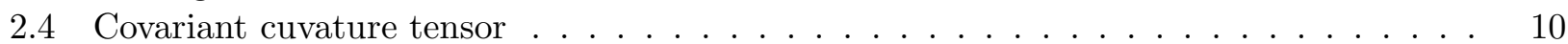

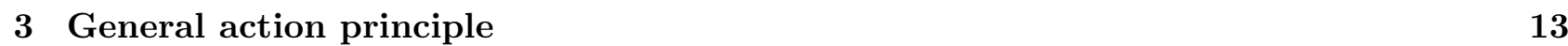

3.1 Gauge invariant action . . . . . . . . . . . . . . . . . 14

3.2 Covariant gauge variation and Bianchi identity . . . . . . . . . . 15

4 Relation to formulation with $\mathcal{E}_{i j} \quad \square$

4.1 Gauge choice . . . . . . . . . . . . . . . . . . . 16

$4.2 O(D, D)$ covariant derivatives and gauge variation $\ldots \ldots \ldots \ldots \ldots$

5 Relation to formulation with $\mathcal{H}^{M N} \quad \mathbf{2 1}$

5.1 Gauge choice and generalized coset formulation . . . . . . . . . . . . . . 21



$5.3 \mathcal{H}$-compatible Christoffel connections . . . . . . . . . . . . . . . . 23

6 Summary and Outlook 25

\begin{tabular}{|l|l|}
\hline A Computational details on the curvature tensor & 27 \\
\hline
\end{tabular}

A.1 Fully contracted curvature tensor without constraints . . . . . . . . . . . . 27



A.3 Scalar curvature in terms of $G L(D) \times G L(D)$ covariant derivatives . . . . . . . . . 30

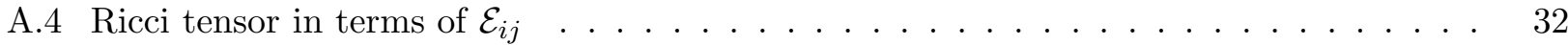

\section{Introduction}

T-duality is one of the simplest and perhaps one of the most intriguing dualities of string theory. It relates the momentum and winding modes of closed string theory on a torus $T^{D}$ via the non-compact duality group $O(D, D)$ (for a review see [1]). It is a fairly natural idea that this duality symmetry can be made manifest upon introducing 'doubled coordinates', both at the level of the world-sheet [2, 3, 4] and at the level of space-time [3, 5]. In other words, in addition to the usual coordinates $x^{i}$ associated to momentum modes one has dual coordinates $\tilde{x}_{i}$ associated to winding modes.

Recently, a formulation of such a 'double field theory' has been found [6, 7, 8, 9, that can be viewed as an $O(D, D)$ covariantization of the low-energy effective space-time action. (For recent papers related to this theory see [10, 11, 12, 13, 14.) The conventional action for the metric $g_{i j}$, the Kalb-Ramond two-form $b_{i j}$ with field strength $H_{i j k}=3 \partial_{[i} b_{j k]}$, and the dilaton $\phi$ is given by

$$
S=\int d x \sqrt{-g} e^{-2 \phi}\left[R+4(\partial \phi)^{2}-\frac{1}{12} H^{2}\right]
$$


The double field theory action is written in terms of the 'non-symmetric metric' $\mathcal{E}_{i j}=g_{i j}+b_{i j}$, which naturally combines the conventional metric and 2 -form, and the dilaton $d$ which is related to the scalar dilaton $\phi$ via the field redefinition $e^{-2 d}=\sqrt{-g} e^{-2 \phi}$. It reads

$$
\begin{aligned}
S=\int d x d \tilde{x} e^{-2 d}[ & -\frac{1}{4} g^{i k} g^{j l} \mathcal{D}^{p} \mathcal{E}_{k l} \mathcal{D}_{p} \mathcal{E}_{i j}+\frac{1}{4} g^{k l}\left(\mathcal{D}^{j} \mathcal{E}_{i k} \mathcal{D}^{i} \mathcal{E}_{j l}+\overline{\mathcal{D}}^{j} \mathcal{E}_{k i} \overline{\mathcal{D}}^{i} \mathcal{E}_{l j}\right) \\
& \left.+\left(\mathcal{D}^{i} d \overline{\mathcal{D}}^{j} \mathcal{E}_{i j}+\overline{\mathcal{D}}^{i} d \mathcal{D}^{j} \mathcal{E}_{j i}\right)+4 \mathcal{D}^{i} d \mathcal{D}_{i} d\right]
\end{aligned}
$$

Here, the calligraphic derivatives are defined by

$$
\mathcal{D}_{i} \equiv \frac{\partial}{\partial x^{i}}-\mathcal{E}_{i k} \frac{\partial}{\partial \tilde{x}_{k}}, \quad \overline{\mathcal{D}}_{i} \equiv \frac{\partial}{\partial x^{i}}+\mathcal{E}_{k i} \frac{\partial}{\partial \tilde{x}_{k}},
$$

and all indices are raised with $g^{i j}$, which is the inverse of the metric $g_{i j}=\frac{1}{2}\left(\mathcal{E}_{i j}+\mathcal{E}_{j i}\right)$. As required, the action (1.2) reduces to (1.1) if the winding derivatives are set to zero [8]. Moreover, it is invariant under the T-duality group $O(D, D)$ whose action on the fields can be written in matrix notation as (generalizing the well-known Buscher rules [15])

$$
\mathcal{E}^{\prime}\left(X^{\prime}\right)=(a \mathcal{E}(X)+b)(c \mathcal{E}(X)+d)^{-1}, \quad d^{\prime}\left(X^{\prime}\right)=d(X), \quad X^{\prime}=h X,
$$

where

$$
h=\left(\begin{array}{ll}
a & b \\
c & d
\end{array}\right) \in O(D, D)
$$

and we have grouped the momentum coordinates $x^{i}$ and the winding coordinates $\tilde{x}_{i}$ into a fundamental $O(D, D)$ vector $X^{M}=\left(\tilde{x}_{i}, x^{i}\right) ! 1$ The action (1.2) is also invariant under a gauge symmetry with a parameter $\xi^{M}=\left(\tilde{\xi}_{i}, \xi^{i}\right)$ that combines the conventional diffeomorphism parameter $\xi^{i}$ with the 1-form gauge parameter $\tilde{\xi}_{i}$ of the 2 -form,

$$
\begin{aligned}
\delta \mathcal{E}_{i j} & =\mathcal{D}_{i} \tilde{\xi}_{j}-\overline{\mathcal{D}}_{j} \tilde{\xi}_{i}+\xi^{M} \partial_{M} \mathcal{E}_{i j}+\mathcal{D}_{i} \xi^{k} \mathcal{E}_{k j}+\overline{\mathcal{D}}_{j} \xi^{k} \mathcal{E}_{i k} \\
\delta d & =\xi^{M} \partial_{M} d-\frac{1}{2} \partial_{M} \xi^{M}
\end{aligned}
$$

and which reduces to the familiar diffeomorphism and 2-form gauge symmetry for $\tilde{\partial}=0$.

The consistency of the action (1.2) requires the constraint that all fields and gauge parameters and all their products are annihilated by the differential operator $\tilde{\partial}^{i} \partial_{i}$. This is a stronger version of the level-matching condition of closed string theory, and will therefore sometimes be referred to as 'the strong constraint'. It takes a manifestly $O(D, D)$ covariant form, upon introducing the $O(D, D)$ invariant metric $\eta$ : We require

$$
\partial^{M} \partial_{M} A=\eta^{M N} \partial_{M} \partial_{N} A=0, \quad \partial^{M} A \partial_{M} B=0, \quad \eta^{M N}=\left(\begin{array}{ll}
0 & 1 \\
1 & 0
\end{array}\right)
$$

\footnotetext{
${ }^{1}$ We use a notation that does not distinguish between compact and non-compact coordinates. In string theory only the compact coordinates should be doubled, and in this case the doubling for the non-compact coordinates is only formal, with a trivial dependence of the fields on these new coordinates. The signature of the duality group $O(D, D)$ applies to the case that the space-time metric $g_{i j}$ is positive definite, but all formulas below extend readily to any other signature.
} 
for all fields and parameters $A, B$. This constraint implies that locally there is always an $O(D, D)$ transformation that rotates into a 'T-duality frame' where the fields depend only on half of the coordinates, for instance the momentum coordinates.

Despite taking a strikingly simple form, the properties of the double field theory action (1.2) are not very transparent. Even though the $O(D, D)$ invariance is well understood (and 'manifest' in the sense that each term is separately invariant [8]), the fields do not transform in linear representations of $O(D, D)$. Moreover, the gauge symmetry is far from being manifest in the formulation (1.2), and therefore a more geometrical understanding is desirable.

More recently, a reformulation of (1.2) has been given in which some of these features are more accessible [9]. It is based on the 'generalized metric'

$$
\mathcal{H}^{M N}=\left(\begin{array}{cc}
g_{i j}-b_{i k} g^{k l} b_{l j} & b_{i k} g^{k j} \\
-g^{i k} b_{k j} & g^{i j}
\end{array}\right),
$$

which combines the metric and 2 -form in such a way that it transforms covariantly under $O(D, D)$ according to its index structure, i.e., in a linear representation as opposed to the non-linear representation of $\mathcal{E}_{i j}$ above. The double field theory action can then be written in the manifestly $O(D, D)$ invariant form

$$
\begin{aligned}
S=\int d x d \tilde{x} e^{-2 d}( & \frac{1}{8} \mathcal{H}^{M N} \partial_{M} \mathcal{H}^{K L} \partial_{N} \mathcal{H}_{K L}-\frac{1}{2} \mathcal{H}^{M N} \partial_{N} \mathcal{H}^{K L} \partial_{L} \mathcal{H}_{M K} \\
& \left.-2 \partial_{M} d \partial_{N} \mathcal{H}^{M N}+4 \mathcal{H}^{M N} \partial_{M} d \partial_{N} d\right)
\end{aligned}
$$

Here, the derivatives $\partial_{M}=\left(\tilde{\partial}^{i}, \partial_{i}\right)$ and $\partial^{M}=\eta^{M N} \partial_{N}=\left(\partial_{i}, \tilde{\partial}^{i}\right)$ transform covariantly under $O(D, D)$. Remarkably, in terms of $\mathcal{H}^{M N}$ the gauge symmetry parameterized by $\xi^{M}$ becomes manifestly $O(D, D)$ invariant,

$$
\delta_{\xi} \mathcal{H}^{M N}=\xi^{P} \partial_{P} \mathcal{H}^{M N}+\left(\partial^{M} \xi_{P}-\partial_{P} \xi^{M}\right) \mathcal{H}^{P N}+\left(\partial^{N} \xi_{P}-\partial_{P} \xi^{N}\right) \mathcal{H}^{M P} .
$$

Here and in the following $O(D, D)$ indices are raised and lowered with $\eta^{M N}$. This form of the gauge transformations naturally suggests a notion of 'generalized Lie derivative', in which each index gives rise to a covariant and a contravariant contribution. In the formulation (1.9) the gauge invariance of the double field theory action can be checked more easily, although it is still non-manifest.

For both formulations presented above the action can actually be written in an Einstein-Hilbert like form with a scalar curvature $\mathcal{R}=\mathcal{R}(\mathcal{E}, d)=\mathcal{R}(\mathcal{H}, d)$ that can be viewed as a function of $d$ and either $\mathcal{E}_{i j}$ or $\mathcal{H}^{M N}$. More precisely, up to boundary terms, (1.2) and (1.9) can be written as

$$
S=\int d x d \tilde{x} e^{-2 d} \mathcal{R}(\mathcal{E}, d)=\int d x d \tilde{x} e^{-2 d} \mathcal{R}(\mathcal{H}, d)
$$

In here, $\mathcal{R}$ transforms as a scalar and $e^{-2 d}$ as a density,

$$
\delta_{\xi} \mathcal{R}=\xi^{M} \partial_{M} \mathcal{R}, \quad \delta_{\xi}\left(e^{-2 d}\right)=\partial_{M}\left(\xi^{M} e^{-2 d}\right),
$$

from which invariance of the action immediately follows. The scalar curvature has, however, only been determined 'by hand' as functions of $d$ and $\mathcal{E}_{i j}$ or $\mathcal{H}^{M N}$, respectively, by requiring the transformation 
behavior (1.12). Again, a more geometrical understanding, in which $\mathcal{R}$ arises from a Riemann-tensorlike object that is manifestly covariant, is desirable.

Prior to these developments Siegel has introduced some time ago a duality-covariant geometrical formalism in a remarkable paper [16] (extending the results of [17]). This formalism is based on a frame-field $e_{A}{ }^{M}$ that carries a flat index $A$ corresponding to a local $G L(D) \times G L(D)$ symmetry. This direct product structure with two independent general linear groups reflects the left-right factorization of closed string theory. The formalism features connections for this local symmetry and covariant curvature tensors. Intriguingly, it has the same transformations under $\xi^{M}$ according to the generalized Lie derivatives as in (1.10), and it requires the same constraint (1.7). Given these and other similarities it is natural to assume that, upon suitable identifications and gauge fixings, the formalism of Siegel is in fact equivalent to the double field theory formulation reviewed above. In this paper we will show that this is indeed the case.

In [9] this relation has already been elaborated at the level of the field content and the symmetry transformations. Here, we go beyond that by relating the curvature scalars appearing in (1.11) to the curvature tensor for the $G L(D) \times G L(D)$ connections of Siegel's formulation. In doing so we believe to both clarify the geometrical meaning of the recent results on double field theory and to give a more explicit and thereby more accessible treatment of Siegel's formalism.

This paper is organized as follows. In sec. 2 we review the $O(D, D)$ covariant generalized Lie derivatives and Siegel's frame-like geometrical formalism. In sec. 3 we discuss the general action principle and derive the Bianchi identities implied by gauge invariance. These two sections are mainly a review of [16] and [9], but also contain novel results, as the manifestly $O(D, D)$ and $G L(D) \times G L(D)$ covariant form (2.59) of the scalar curvature. The main results of this paper are given in sec. 4 and 5 , where we relate the frame formalism to the explicit formulations in terms of $\mathcal{E}_{i j}$ and $\mathcal{H}^{M N}$. Specifically, in sec. 4 we show the equivalence of the scalar curvature and the corresponding scalar found in [8], and relate in particular ' $O(D, D)$ covariant derivatives' introduced there to the $G L(D) \times G L(D)$ connections. In sec. 5 we give an independent proof of the equivalence of the curvature scalars in the formulation with $\mathcal{H}^{M N}$ given in [9]. We close with a summary and outlook in sec. 6. Some technically involved calculations related to the curvature tensor can be found in the appendix.

\section{Geometrical frame formalism}

In this section we first review the novel gauge transformations parametrized by $\xi^{M}$ and the associated C-bracket. Next we introduce frame fields which are subject to the tangent space symmetry $G L(D) \times$ $G L(D)$ together with connections for this symmetry. Finally, a covariant curvature tensor is discussed.

\subsection{Generalized Lie derivatives, Courant bracket and frame fields}

The generalized Lie derivative is defined for tensors with an arbitrary number of upper and lower $O(D, D)$ indices by the straightforward extension of

$$
\widehat{\mathcal{L}}_{\xi} A_{M}^{N} \equiv \xi^{P} \partial_{P} A_{M}^{N}+\left(\partial_{M} \xi^{P}-\partial^{P} \xi_{M}\right) A_{P}^{N}+\left(\partial^{N} \xi_{P}-\partial_{P} \xi^{N}\right) A_{M}^{P}
$$


With this definition the gauge transformation (1.10) simply reduces to

$$
\delta_{\xi} \mathcal{H}^{M N}=\widehat{\mathcal{L}}_{\xi} \mathcal{H}^{M N}
$$

In general we will refer to $O(D, D)$ tensors that transform according to the generalized Lie derivative under gauge transformations parameterized by $\xi^{M}$ as 'generalized tensors' or as transforming covariantly under $\xi^{M}$.

An important consistency property of this formalism is that the $O(D, D)$ invariant metric that is used in (2.1) to raise and lower indices has vanishing generalized Lie derivative,

$$
\widehat{\mathcal{L}}_{\xi} \eta^{M N}=\xi^{P} \partial_{P} \eta^{M N}-\partial^{N} \xi^{M}-\partial^{M} \xi^{N}+\partial^{N} \xi^{M}+\partial^{M} \xi^{N}=0 .
$$

Accordingly, in this formalism it is consistent to have this constant tensor with two upper or two lower 'curved' or 'world' indices.

The closure of the gauge transformations spanned by $\xi^{M}$ or, equivalently, the algebra of generalized Lie derivatives can be straightforwardly determined in this formulation and is governed by the ' $\mathrm{C}$ bracket',

$$
\left[\widehat{\mathcal{L}}_{\xi_{1}}, \widehat{\mathcal{L}}_{\xi_{2}}\right]=-\widehat{\mathcal{L}}_{\left[\xi_{1}, \xi_{2}\right]_{\mathrm{C}}}
$$

where

$$
\left[\xi_{1}, \xi_{2}\right]_{\mathrm{C}}^{M} \equiv \xi_{1}^{N} \partial_{N} \xi_{2}^{M}-\frac{1}{2} \xi_{1}^{P} \partial^{M} \xi_{2 P}-(1 \leftrightarrow 2) .
$$

This bracket is the $O(D, D)$ covariant double field theory extension of the Courant bracket of generalized geometry [18, 19, 20], as has been shown in [7]. An important property that will be used later is that the C-bracket of two generalized vectors is again a generalized vector. In order to verify this let $X^{M}$ and $Y^{M}$ be transforming as $\delta_{\xi} X^{M}=\widehat{\mathcal{L}}_{\xi} X^{M}$ and $\delta_{\xi} Y^{M}=\widehat{\mathcal{L}}_{\xi} Y^{M}$, respectively. For the computation of the gauge variation of their C-bracket it is useful to keep in mind that the variation of an $O(D, D)$ invariant expression automatically combines into the covariant terms according to the generalized Lie derivative and into non-covariant terms that originate exclusively from the variation of partial derivatives. Thus, we find

$$
\begin{aligned}
\delta_{\xi}[X, Y]_{\mathrm{C}}^{M}= & \delta_{\xi}\left(X^{N} \partial_{N} Y^{M}-\frac{1}{2} X^{P} \partial^{M} Y_{P}-(X \leftrightarrow Y)\right) \\
= & \widehat{\mathcal{L}}_{\xi}[X, Y]_{\mathrm{C}}^{M} \\
& +X^{N} \partial_{N}\left(\partial^{M} \xi_{K}-\partial_{K} \xi^{M}\right) Y^{K}-\frac{1}{2} X^{P} \partial^{M}\left(\partial_{P} \xi^{K}-\partial^{K} \xi_{P}\right) Y_{K}-(X \leftrightarrow Y) \\
= & \widehat{\mathcal{L}}_{\xi}[X, Y]_{\mathrm{C}}^{M},
\end{aligned}
$$

where the cancelation of the non-covariant terms easily follows from the antisymmetry in $X$ and $Y$. This establishes the covariance of the C-bracket. As the variation in the first line can also be written as $[\delta X, Y]_{\mathrm{C}}+[X, \delta Y]_{\mathrm{C}}$, this covariance property of the C-bracket can be put more compactly as

$$
\widehat{\mathcal{L}}_{\xi}[X, Y]_{\mathrm{C}}=\left[\widehat{\mathcal{L}}_{\xi} X, Y\right]_{\mathrm{C}}+\left[X, \widehat{\mathcal{L}}_{\xi} Y\right]_{\mathrm{C}},
$$

which is the analogue of the invariance of the Lie bracket under the usual Lie derivative. 
In general, partial derivatives of generalized tensors are not generalized tensors. An exception is a generalized scalar $S$ which according to (2.1) simply transforms as

$$
\delta_{\xi} S=\widehat{\mathcal{L}}_{\xi} S=\xi^{P} \partial_{P} S .
$$

Therefore, its partial derivative transforms as

$$
\delta_{\xi}\left(\partial_{M} S\right)=\partial_{M}\left(\xi^{P} \partial_{P} S\right)=\xi^{P} \partial_{P}\left(\partial_{M} S\right)+\left(\partial_{M} \xi^{P}-\partial^{P} \xi_{M}\right) \partial_{P} S \equiv \widehat{\mathcal{L}}_{\xi}\left(\partial_{M} S\right),
$$

where in the second equality we were allowed to add the third term because it is zero by the constraint (1.7). Thus, $\partial_{M} S$ transforms covariantly, i.e., as a generalized covariant tensor. This covariant transformation behavior does not hold for partial derivatives of higher tensors, not even for antisymmetrized combinations like $\partial_{[M} V_{N]}$ - in contrast to conventional diffeomorphisms.

In the following we will introduce a frame field which allows to convert arbitrary tensors from 'world'-tensors into 'tangent space'-tensors and thereby into scalars under $\xi^{M}$. Specifically, following Siegel [16] we introduce a frame field $e_{A}{ }^{M}$, which is a generalized vector and has a flat index $A$ corresponding to a local $G L(D) \times G L(D)$ symmetry, i.e. 2

$$
e_{A}^{M}=\left(\begin{array}{cc}
e_{a i} & e_{a}^{i} \\
e_{\bar{a} i} & e_{\bar{a}}^{i}
\end{array}\right)
$$

We assume this vielbein to be invertible and denote the inverse by $e_{M}{ }^{A}$. In (2.10) we used the splitting $M=\left({ }_{i},{ }^{i}\right)$ of the $O(D, D)$ index and $A=(a, \bar{a})$ is the $G L(D) \times G L(D)$ index. Given the $O(D, D)$ invariant metric $\eta_{M N}$ we can build an $X$-dependent 'tangent space' metric of signature $(D, D)$,

$$
\mathcal{G}_{A B}=e_{A}^{M} e_{B}^{N} \eta_{M N}
$$

with inverse $\mathcal{G}^{A B}=\eta^{M N} e_{M}{ }^{A} e_{N}{ }^{B}$, which will be used to raise and lower flat indices. The raising and lowering of world indices with $\eta$ and of flat indices with $\mathcal{G}$ is consistent with inverting the frame field (2.10) in that

$$
e_{M}^{A}=\eta_{M N} \mathcal{G}^{A B} e_{B}{ }^{N} \quad \Rightarrow \quad e_{M}^{A} e_{A}{ }^{N}=\delta_{M}{ }^{N}
$$

as follows from the definition (2.11). In order for $e_{A}{ }^{M}$ to describe only the physical degrees of freedom it turns out to be necessary to impose the $G L(D) \times G L(D)$ covariant constraint

$$
\mathcal{G}_{a \bar{b}}=0 \quad \Leftrightarrow \quad e_{(a}^{i} e_{\bar{b}) i}=0
$$

which is related to the left-right factorization of closed string theory [16] 3

Using the frame field one can introduce a 'flattened' derivative $e_{A}$, defined by

$$
e_{A} \equiv e_{A}^{M} \partial_{M}
$$

\footnotetext{
${ }^{2}$ We note that our conventions for the frame field differ from those in 9 . (c.f. eq. (5.12)) in order to be more in line with 16 .

${ }^{3} \mathrm{An}$ alternative motivation of this constraint starting from generalized geometry and the generalized metric $\mathcal{H}$ has been given in 9], c.f. the discussion after eq. (5.1) below.
} 
We note that the strong constraint (1.7) takes the following form in terms of flat indices,

$$
e^{A} X e_{A} Y=\mathcal{G}^{A B} e_{A}^{M} e_{B}^{N} \partial_{M} X \partial_{N} Y=\eta^{M N} \partial_{M} X \partial_{N} Y=0
$$

for arbitrary functions $X$ and $Y$. Due to the covariance of the partial derivative of a generalized scalar discussed above, the action of $e_{A}$ on an arbitrary tensor with only flat indices, $e_{A} X_{B C \ldots}$, is covariant under $\xi^{M}$ transformations. Of course, it will not be covariant under the local frame rotations, and so covariant derivatives have to be introduced. Thereby, the problem of defining derivative operations that are covariant under generalized diffeomorphisms parameterized by $\xi^{M}$ has been converted to the problem of introducing covariant derivatives and connections for the $G L(D) \times G L(D)$ tangent space symmetry, to which we turn now.

\section{$2.2 G L(D) \times G L(D)$ connections and constraints}

We define the infinitesimal local $G L(D) \times G L(D)$ transformations to be

$$
\delta_{\Lambda} V_{A}=\Lambda_{A}^{B} V_{B}, \quad \delta_{\Lambda} V^{A}=-\Lambda_{B}^{A} V^{B}
$$

and analogously for tensors with an arbitrary number of upper and lower indices. Since we are dealing with $G L(D) \times G L(D)$, the non-vanishing parameters are $\Lambda_{a}{ }^{b}$ and $\Lambda_{\bar{a}}{ }^{\bar{b}}$. Covariant derivatives with flattened indices are given by

$$
\nabla_{A} V_{B}=e_{A} V_{B}+\omega_{A B}{ }^{C} V_{C}, \quad \nabla_{A} V^{B}=e_{A} V^{B}-\omega_{A C}{ }^{B} V^{C},
$$

where we have introduced connections $\omega_{A B}{ }^{C}$. Again, since we are dealing with gauge group $G L(D) \times$ $G L(D)$ the only non-vanishing components of the connections are

$$
\omega_{A B}^{C}: \quad \omega_{A b}^{c}, \omega_{A \bar{b}}^{\bar{c}} .
$$

Moreover, the constraint (2.13) implies that the same holds for connections with all indices lowered. We will frequently make use of the fact that components like $\omega_{a b}{ }^{\bar{c}}$ and $\omega_{a b \bar{c}}$ vanish. We require that the connections transform under $\xi^{M}$ as scalars and therefore, as discussed above, the covariant derivatives (2.17) transform as scalars, too. They transform also covariantly under $G L(D) \times G L(D)$ if we require that the $\omega_{A B}{ }^{C}$ transform as connections, i.e.,

$$
\delta \omega_{A a}{ }^{b}=-\nabla_{A} \Lambda_{a}^{b}+\Lambda_{A}{ }^{B} \omega_{B a}{ }^{b}, \quad \nabla_{A} \Lambda_{a}^{b}=e_{A} \Lambda_{a}^{b}+\omega_{A a}{ }^{c} \Lambda_{c}^{b}-\omega_{A c}{ }^{b} \Lambda_{a}^{c},
$$

and analogously for barred indices. We note that the additional term in $\delta \omega_{A a}{ }^{b}$ as compared to the familiar transformation rule for a Yang-Mills gauge potential is due to the conversion of the 1-form index into a flat one.

Next we have to impose covariant constraints that allow us to solve for (part of) the connections in terms of the physical fields. A natural starting point is the C-bracket governing the gauge algebra. In ordinary Riemannian geometry the torsion constraint of the Levi-Civita connection implies that in the Lie bracket of two vector fields the partial derivatives can be replaced by covariant derivatives. In the double field theory context the Lie bracket is replaced by the C-bracket in that only the latter 
transforms covariantly under generalized diffeomorphisms. Since we are dealing here with flattened derivatives it is thus natural to define a torsion tensor in such a way that it vanishes if and only if in the C-bracket with flattened parameter $\xi_{12}^{A}=\left[\xi_{1}, \xi_{2}\right]_{\mathrm{C}}^{M} e_{M}^{A}$ the partial derivatives are replaced by $G L(D) \times G L(D)$ covariant derivatives, i.e.,

$$
\xi_{12}^{A}=\xi_{1}^{B} \nabla_{B} \xi_{2}^{A}-\frac{1}{2} \xi_{1 B} \nabla^{A} \xi_{2}^{B}-(1 \leftrightarrow 2)+\xi_{1}^{B} \xi_{2}^{C} \mathcal{T}_{B C}{ }^{A} .
$$

This leads td 4

$$
\mathcal{T}_{A B}^{C}=\Omega_{A B}^{C}+2\left(\omega_{[A B]}^{C}+\frac{1}{2} \omega^{C}{ }_{[A B]}\right)
$$

where

$$
\Omega_{A B}^{C}=2\left(f_{[A B]}^{C}+\frac{1}{2} f^{C}{ }_{[A B]}\right), \quad f_{A B C} \equiv\left(e_{A} e_{B}{ }^{M}\right) e_{C M} .
$$

Another more covariant form of the torsion tensor is

$$
\mathcal{T}_{A B}{ }^{C}=2\left(e_{N}{ }^{C} \nabla_{[A} e_{B]}{ }^{N}-\frac{1}{2} e_{N[A} \nabla^{C} e_{B]}{ }^{N}\right)
$$

We note here that the torsion tensor defined like this does not coincide with the usual definition via the commutator of covariant derivatives. We will return to this issue below.

The $\Omega_{A B}{ }^{C}$ introduced above can be seen as generalized 'coefficients of anholonomy' in that

$$
\left[e_{A}, e_{B}\right]=\Omega_{A B}{ }^{C} e_{C} .
$$

To be more precise, the first term in (2.22) proportional to $f_{[A B]}{ }^{C}$ corresponds to the usual coefficients of anholonomy, while the second term drops out in the equation (2.24) by virtue of the constraint (2.15). The full $\Omega_{A B}{ }^{C}$ are obtained unambiguously from the C-bracket,

$$
\left[e_{A}, e_{B}\right]_{\mathrm{C}}^{M}=\Omega_{A B}^{C} e_{C}^{M}
$$

This can be verified by inserting the components $e_{A}^{M}$ into the definition (2.5). These generalized coefficients of anholonomy, as opposed to the usual ones and the $f_{A B C}$, are fully covariant under $\xi^{M}$ transformations. This follows directly from (2.25) and the fact that the C-bracket transforms covariantly. Since the $\omega_{A B}{ }^{C}$ are generalized scalars it follows from (2.21) that the torsion tensor transforms covariantly under $\xi^{M}$, while its covariance under $G L(D) \times G L(D)$ is manifest in the form (2.23). Alternatively, this covariance can be inferred from the defining equation (2.20) and the covariance of the C-bracket. In total, imposing the torsion constraint

$$
\mathcal{T}_{A B}{ }^{C}=0
$$

is consistent with all symmetries.

Next, we impose the 'metricity condition' that the metric $\mathcal{G}_{A B}$ is covariantly constant [16],

$$
\nabla_{A} \mathcal{G}_{B C}=0 \quad \Leftrightarrow \quad e_{A} \mathcal{G}_{B C}+2 \omega_{A(B C)}=0 .
$$

\footnotetext{
${ }^{4}$ In this paper we employ the convention that symmetrization and anti-symmetrization involves the combinatorial factor, e.g., $X_{[a b]}=\frac{1}{2}\left(X_{a b}-X_{b a}\right)$. In some formulas this leads to numerical factors that are different from those in [16].
} 
We recall that indices (here on $\omega$ ) are lowered with $\mathcal{G}_{A B}$.

Finally, we impose a constraint that allows for partial integrations in an action using the covariant derivatives [16]. Specifically, as actions are defined with the density $e^{-2 d}$, we require

$$
\int e^{-2 d} V \nabla_{A} V^{A}=-\int e^{-2 d} V^{A} \nabla_{A} V=-\int e^{-2 d} V^{A} e_{A} V
$$

for arbitrary $V$ and $V^{A}$. The consistency of this and the previous constraints will be confirmed in the next subsection by providing the explicit solutions.

\subsection{Solving the constraints}

We solve now the above constraints and show their mutual consistency. First, the metricity condition (2.27) can be trivially solved,

$$
\omega_{A(B C)}=-\frac{1}{2} e_{A} \mathcal{G}_{B C}=-f_{A(B C)},
$$

and determines the part symmetric in the 'group indices' of $\omega_{A B C}$ completely.

We turn next to the torsion constraint (2.26), which reads explicitly

$$
\Omega_{A B C}=\omega_{B A C}+\frac{1}{2} \omega_{C B A}-\omega_{A B C}-\frac{1}{2} \omega_{C A B} .
$$

To simplify this further we decompose $\omega_{A B C}$ into a part which is antisymmetric in its last two indices and into a part which is symmetric in its last two indices,

$$
\begin{aligned}
\Omega_{A B C} & =\omega_{B[A C]}+\omega_{B(A C)}+\frac{1}{2}\left(\omega_{C[B A]}+\omega_{C(B A)}\right)-\omega_{A[B C]}-\omega_{A(B C)}-\frac{1}{2}\left(\omega_{C[A B]}+\omega_{C(A B)}\right) \\
& =3 \omega_{[B A C]}+\omega_{B(A C)}-\omega_{A(B C)} .
\end{aligned}
$$

It follows that the completely antisymmetric part is fully determined,

$$
\omega_{[A B C]}=-\frac{1}{3} \Omega_{[A B C]}=-f_{[A B C]} .
$$

In order to gain further insights from (2.31) we decompose the $G L(D) \times G L(D)$ indices and use that only $\omega_{A a b}$ and $\omega_{A \bar{a} \bar{b}}$ are non-zero. This leads to

$$
\Omega_{a \bar{b} \bar{c}}=3 \omega_{[\bar{b} a \bar{c}]}+\omega_{\bar{b}(a \bar{c})}-\omega_{a(\bar{b} \bar{c})}=-\omega_{a[\bar{b} \bar{c}]}-\omega_{a(\bar{b} \bar{c})}=-\omega_{a \bar{b} \bar{c}},
$$

and similarly for the opposite index structure. Thus, in total

$$
\omega_{a \bar{b} \bar{c}}=-\Omega_{a \bar{b} \bar{c}}, \quad \omega_{\bar{a} b c}=-\Omega_{\bar{a} b c} .
$$

These equations determine, in particular, symmetric parts as $\omega_{a(\bar{b} \bar{c})}$ which were already given by (2.29). They are, however, consistent as can be confirmed by an explicit computation using (2.22),

$$
\omega_{a(\bar{b} \bar{c})}=-\Omega_{a(\bar{b} \bar{c})}=-\frac{1}{2} e_{a} \mathcal{G}_{\bar{b} \bar{c}}
$$

and similarly for the opposite index structure. 
Finally, we solve the constraint (2.28), which after integration by parts reads explicitly

$$
\begin{aligned}
\int e^{-2 d} V \nabla_{A} V^{A} & =\int e^{-2 d} V\left(e_{A}{ }^{M} \partial_{M} V^{A}-\omega_{A B}{ }^{A} V^{B}\right) \\
& =-\int e^{-2 d} V^{A}\left(e_{A}^{M} \partial_{M} V+e^{2 d} V \partial_{M}\left(e_{A}{ }^{M} e^{-2 d}\right)+V \omega_{B A}{ }^{B}\right) .
\end{aligned}
$$

From this we read off

$$
\omega_{B A}^{B}=-\tilde{\Omega}_{A} \equiv-e^{2 d} \partial_{M}\left(e_{A}^{M} e^{-2 d}\right)=-\partial_{M} e_{A}^{M}+2 e_{A} d,
$$

where we introduced $\tilde{\Omega}_{A}$ for notational convenience. This constraint can be interpreted as setting the following 'new torsion' to zero,

$$
\tilde{T}_{A}=\partial_{M} e_{A}^{M}+\omega_{B A}^{B}-2 e_{A} d=0,
$$

which yields for a scalar $S$

$$
\nabla_{A} \nabla^{A} S=-\tilde{T}_{A} \nabla^{A} S=0
$$

where the first equality follows by virtue of the strong constraint (2.15).

We conclude this section by summarizing which connections are determined by the above constraints (2.26), (2.27) and (2.28). First, the 'off-diagonal' components $\omega_{\bar{a} b c}$ and $\omega_{a \bar{b} \bar{c}}$ are completely determined according to (2.34). For the 'diagonal' components $\omega_{a b c}$ and $\omega_{\bar{a} \bar{b} \bar{c}}$ the parts symmetric in the last two indices are fully determined by (2.29). Therefore, it is sufficient for the remaining components to focus on the part antisymmetric in the last two indices, whose irreducible parts, say for $\omega_{a b c}$, are given by the following tensor product

$$
\omega_{a[b c]}: \quad \square \otimes \square=\square \oplus \square,
$$

where the Young tableaux refer to the left $G L(D)$ group. In here, the completely antisymmetric

part $\omega_{[a b c]}$ is determined by (2.32). For the 'mixed-Young tableaux' representation on the right-hand side of (2.40) the trace parts are determined by (2.37) in terms of the dilaton, leaving precisely the trace-free part of this $(2,1)$ representation as the undetermined connections. Its dimension is given by $\frac{1}{3} D(D+2)(D-2)$ and therefore, taking the right $G L(D)$ into account, the number of undetermined components is twice this value. That not all components are determined by the above constraints limits the extent to which invariant curvatures can be constructed out of the physical fields, which will be discussed in the next subsection.

\subsection{Covariant cuvature tensor}

Let us now turn to the construction of invariant curvature tensors for the $G L(D) \times G L(D)$ connections. In general, given covariant derivatives one can define curvatures through their commutator, say, acting on $V_{C}$,

$$
\left[\nabla_{A}, \nabla_{B}\right] V_{C}=T_{A B}^{D} \nabla_{D} V_{C}+R_{A B C}^{D} V_{D}
$$


This leads to the standard expressions

$$
\begin{aligned}
T_{A B}{ }^{C} & =\Omega_{A B}^{C}+2 \omega_{[A B]}{ }^{C} \\
R_{A B C}{ }^{D} & =e_{A} \omega_{B C}^{D}-e_{B} \omega_{A C}{ }^{D}+\omega_{A C}{ }^{E} \omega_{B E}{ }^{D}-\omega_{B C}{ }^{E} \omega_{A E}{ }^{D}-\Omega_{A B}{ }^{E} \omega_{E C}{ }^{D} .
\end{aligned}
$$

We note that the torsion tensor $T_{A B}{ }^{C}$ defined like this does not coincide with the torsion $\mathcal{T}_{A B}{ }^{C}$ defined earlier in (2.21). Given the modification of the $\xi^{M}$ gauge transformations as compared to the standard diffeomorphisms it was, however, only consistent to set $\mathcal{T}_{A B}{ }^{C}=0$. We conclude that the conventional torsion is necessarily non-zero when imposing (2.26). More precisely, comparing (2.21) with (2.42) one finds the non-vanishing torsion

$$
T_{A B}^{C}=-\omega^{C}{ }_{[A B]} .
$$

Consequently, the commutator (2.41) of covariant derivatives reads

$$
\left[\nabla_{A}, \nabla_{B}\right] V_{C}=-\omega^{D}{ }_{[A B]} \nabla_{D} V_{C}+R_{A B C}{ }^{D} V_{D}
$$

An immediate consequence is that $R_{A B C}{ }^{D}$ as defined in (2.43) cannot be fully covariant with respect to $G L(D) \times G L(D)$, because the left-hand side of (2.45) is manifestly covariant but the right-hand side contains a bare gauge field.

At this stage a comment is in order regarding the non-covariance of the curvature tensor $R$, because formally it coincides with a conventional field strength (with flattened indices) that would be covariant with respect to (frame-)transformations of an arbitrary gauge group. The subtlety here is that the generalized coefficients of anholonomy $\Omega_{A B}{ }^{C}$ defined in (2.22) rather than the conventional ones appear in the last term of (2.43). Actually, eq. (2.41) does not determine whether (2.42) should contain the generalized coefficients of anholonomy or the conventional ones, for in the commutator (2.24) the difference between the two is immaterial by virtue of the strong constraint (2.15), as we saw above. The choice made here is covariant under $\xi^{M}$ gauge transformations, at the cost of violating the $G L(D) \times G L(D)$ covariance.

Next, we compute the failure of covariance in order to repair it in a second step, following [16]. The non-covariance can be inferred from the variation of the bare gauge field in (2.45),

$$
\begin{aligned}
-\delta_{\Lambda} \omega^{D}{ }_{[A B]} \nabla_{D} V_{C} & =e^{D} \Lambda_{[A}^{F} \mathcal{G}_{B] F}\left(e_{D} V_{C}+\omega_{D C}{ }^{E} V_{E}\right)+\text { covariant terms } \\
& =e^{E} \Lambda_{[A}^{F} \mathcal{G}_{B] F} \omega_{E C}{ }^{D} V_{D}+\text { covariant terms }
\end{aligned}
$$

where in the second line we relabeled indices and used the constraint (2.15). Since the non-covariance must be compensated by a non-covariant variation of $R$ we conclude

$$
\delta_{\Lambda} R_{A B C}{ }^{D}=-\left(e^{E} \Lambda_{[A}^{F}\right) \mathcal{G}_{B] F} \omega_{E C}^{D}+\text { covariant terms }
$$

which can also be checked directly with (2.43). We define now a modified curvature tensor [16]

$$
\mathcal{R}_{A B C D}^{\prime} \equiv \frac{1}{2}\left[R_{A B C D}+R_{C D A B}\right]+\frac{1}{4}\left[\omega_{E C D} \omega^{E}{ }_{B A}+\omega_{E A B} \omega^{E}{ }_{D C}\right]
$$


Using (2.47) and (2.19) it is straightforward to compute the non-covariant terms in its gauge variation,

$$
\begin{aligned}
\delta_{\Lambda} \mathcal{R}_{A B C D}^{\prime} & =-\frac{1}{2}\left(e^{E} \Lambda_{A}{ }^{F}\right) \mathcal{G}_{B F} \omega_{E(C D)}-\frac{1}{2}\left(e^{E} \Lambda_{C}{ }^{F}\right) \mathcal{G}_{D F} \omega_{E(A B)} \\
& =\frac{1}{4}\left(e^{E} \Lambda_{A}{ }^{F}\right) \mathcal{G}_{B F} e_{E} \mathcal{G}_{C D}+\frac{1}{4}\left(e^{E} \Lambda_{C}{ }^{F}\right) \mathcal{G}_{D F} e_{E} \mathcal{G}_{A B}=0
\end{aligned}
$$

where we used (2.29) and the constraint (2.15). Thus, $\mathcal{R}^{\prime}$ transforms covariantly under all symmetries. Since the proof of covariance requires the use of the metricity condition, $\mathcal{R}^{\prime}$ transforms only covariantly after imposing this constraint. This can, however, be relaxed by adding further terms that are zero upon imposing the constraints. Specifically, defining

$$
\mathcal{R}_{A B C D}=\mathcal{R}_{A B C D}^{\prime}-\frac{1}{4} \omega_{E C D} \nabla^{E} \mathcal{G}_{A B}-\frac{1}{4} \omega_{E A B} \nabla^{E} \mathcal{G}_{C D}
$$

we obtain a tensor that is fully covariant independently of the constraints. In the remainder of this paper we will assume that all constraints are satisfied, for which $\mathcal{R}=\mathcal{R}^{\prime}$, unless stated differently.

In the rest of this section we examine the symmetry properties and identities of $\mathcal{R}_{A B C D}$. We start with the original curvature $R_{A B C}{ }^{D}$. It is manifestly antisymmetric in its first two indices. It is not manifestly antisymmetric in its last two indices, but this follows nevertheless as a consequence of the metricity condition. To see this we write (2.41) acting on a vector with an upper index,

$$
\left[\nabla_{A}, \nabla_{B}\right] V^{D}=T_{A B}^{E} \nabla_{E} V^{D}-R_{A B C}{ }^{D} V^{C} .
$$

By the covariant constancy of the metric this is related to the commutator acting on a vector with a lower index,

$$
\mathcal{G}^{C D}\left[\nabla_{A}, \nabla_{B}\right] V_{C}=\mathcal{G}^{C D}\left(T_{A B}{ }^{E} \nabla_{E} V_{C}+R_{A B C}{ }^{E} V_{E}\right)=T_{A B}{ }^{E} \nabla_{E} V^{D}+R_{A B}{ }^{D} V^{C}
$$

Comparison with (2.51) then implies the antisymmetry in the last two indices. Summarizing, $R$ has the following symmetries

$$
R_{A B C D}=-R_{B A C D}=-R_{A B D C}
$$

Moreover, since the gauge group is $G L(D) \times G L(D)$ the 'off-diagonal' components in the group indices of $R_{A B C}{ }^{D}$, i.e., in the last two indices, are zero,

$$
R_{A B c \bar{d}}=R_{A B \bar{c} d}=0
$$

corresponding to the fact that the only non-zero connections are (2.18).

Next, we turn to the symmetry properties of $\mathcal{R}$. In general, the correction terms proportional to the connections in (2.48) have no specific symmetry. If we focus on off-diagonal $G L(D) \times G L(D)$ components, however, these extra terms vanish, see (2.18), and so the antisymmetry properties of $R$ elevate to $\mathcal{R}$. For instance,

$$
\mathcal{R}_{a b \bar{c} d}=\frac{1}{2}\left[R_{a b \bar{c} d}+R_{\bar{c} d a b}\right]=\frac{1}{2} R_{\bar{c} d a b}=-\frac{1}{2} R_{\bar{c} d b a}=-\mathcal{R}_{b a \bar{c} d} .
$$

The same conclusion applies to all other components that have precisely three unbarred or three barred indices. 
We close this section with a brief discussion of a curvature scalar that will be used in the next section to define an action. The scalar that is obtained by tracing $\mathcal{R}$ turns out to be zero by virtue of the constraints. Specifically, prior to imposing any constraints, one can prove (see appendix A1) that 5

$$
\mathcal{R}_{A B}{ }^{A B}=2 \nabla_{A} \tilde{T}^{A}+\tilde{T}_{A}^{2}+\nabla_{A} \nabla_{B} \mathcal{G}^{A B}-\frac{1}{6} \mathcal{T}_{[A B C]}{ }^{2}-\frac{3}{8} \nabla_{(A} \mathcal{G}_{B C)}{ }^{2}
$$

Each term vanishes separately after imposing the constraints, and therefore

$$
0=\mathcal{R}_{A B}{ }^{A B}=\mathcal{R}_{a b}^{a b}+\mathcal{R}_{\bar{a} \bar{b}}^{\bar{a} \bar{b}}
$$

Thus, there is a unique way to define a (non-vanishing) scalar,

$$
\mathcal{R}:=-\frac{1}{2} \mathcal{R}_{a b}^{a b}=\frac{1}{2} \mathcal{R}_{\bar{a} \bar{b}} \overline{\bar{a}} \bar{b},
$$

which by construction is a scalar under $\xi^{M}$ transformations and $G L(D) \times G L(D)$.

An expression for $\mathcal{R}$ that makes the invariance under $O(D, D)$ and frame transformations manifest is the following,

$$
\begin{aligned}
\mathcal{R}= & -\left(\nabla^{a} \nabla_{a} d-\nabla^{\bar{a}} \nabla_{\bar{a}} d\right)-\frac{1}{2}\left(\nabla^{a}\left(e_{a}{ }^{M} \nabla^{\bar{b}} e_{\bar{b} M}\right)-\nabla^{\bar{a}}\left(e_{\bar{a}}{ }^{M} \nabla^{b} e_{b M}\right)\right) \\
& -\frac{1}{4}\left(e_{a}{ }^{M} \nabla^{b} e^{\bar{c}}{ }_{M} e^{a N} \nabla_{b} e_{\bar{c} N}-e_{\bar{a}}{ }^{M} \nabla^{\bar{b}} e^{c}{ }_{M} e^{\bar{a} N} \nabla_{\bar{b}} e_{c N}\right) \\
& +\frac{1}{2}\left(e_{\bar{c}}{ }^{M} \nabla_{a} e_{b M} e^{\bar{c} N} \nabla^{b} e^{a}{ }_{N}-e_{c}{ }^{M} \nabla_{\bar{a}} e_{\bar{b} M} e^{c N} \nabla^{\bar{b}} e^{\bar{a}}{ }_{N}\right) \\
& -\left(\nabla^{a} d\left(e_{a}{ }^{M} \nabla^{\bar{b}} e_{\bar{b} M}\right)-\nabla^{\bar{a}} d\left(e_{\bar{a}}{ }^{M} \nabla^{b} e_{b M}\right)\right)-\left(\nabla^{a} d \nabla_{a} d-\nabla^{\bar{a}} d \nabla_{\bar{a}} d\right),
\end{aligned}
$$

which will be verified in appendix A3.

It is not manifest either from the definition (2.58) or the explicit form (2.59) that the scalar curvature depends only on the connection components that have been determined by the constraints. A somewhat lengthy calculation shows, however, that $\mathcal{R}$ can be written as 6

$$
\mathcal{R}=e_{a} \tilde{\Omega}^{a}+\frac{1}{2} \tilde{\Omega}_{a}^{2}+\frac{1}{2} e_{a} e_{b} \mathcal{G}^{a b}-\frac{1}{4} \Omega_{a b \bar{c}}{ }^{2}-\frac{1}{12} \Omega_{[a b c]}{ }^{2}+\frac{1}{8} e^{a} \mathcal{G}^{b c} e_{b} \mathcal{G}_{a c},
$$

as we show in appendix A2. This proves that $\mathcal{R}$ is a well-defined function of the physical fields.

\section{General action principle}

In this section we briefly introduce an Einstein-Hilbert like action principle based on the invariant curvature scalar discussed above, and derive Bianchi identities from its gauge invariance.

\footnotetext{
${ }^{5}$ We note that this expression differs from that in sec. VIII of [16] because of different conventions regarding symmetrization. Moreover, it differs by an overall factor and a relative factor in the last term.

${ }^{6}$ Again, this expression differs from that given in sec. VIII of [16] because of different conventions regarding antisymmetrization, but it also corrects a typo in the fourth term.
} 


\subsection{Gauge invariant action}

Having the scalar $\mathcal{R}$ at our disposal we can define the following action principle

$$
S=\int d x d \tilde{x} e^{-2 d} \mathcal{R}
$$

which, by virtue of $e^{-2 d}$ transforming as a density, is manifestly invariant under all symmetries.

There are a number of conclusions that can be derived from this invariance. First, the variation with respect to $d$ has to be a $G L(D) \times G L(D)$ invariant scalar and therefore it must be proportional to $\mathcal{R}$ defined in (2.58) [16], which conclusion agrees with the results of [8, 9], as we will show below. Second, the general variation with respect to $e_{A}^{M}$ is non-trivial only in its off-diagonal component, in the following sense. Introducing a variation with both indices flat,

$$
\Delta e_{A B}:=e_{B}^{M} \delta e_{A M}
$$

we infer that the $G L(D) \times G L(D)$ transformations (2.16) read

$$
\Delta e_{A B}=e_{B}{ }^{M} \Lambda_{A}{ }^{C} e_{C M}=\Lambda_{A}{ }^{C} \mathcal{G}_{B C}=\Lambda_{A B} .
$$

By the constraint (2.13) this implies

$$
\Delta e_{a b}=\Lambda_{a b}, \quad \Delta e_{\bar{a} \bar{b}}=\Lambda_{\bar{a} \bar{b}}, \quad \Delta e_{a \bar{b}}=-\Delta e_{\bar{b} a}=0 .
$$

Consequently, the local $G L(D) \times G L(D)$ symmetry of the action implies the 'Bianchi identity' that the diagonal parts of the field equations obtained by variation with respect to $\Delta e_{a b}$ and $\Delta e_{\bar{a} \bar{b}}$ vanish identically. Thus, the only non-trivial part of the field equation is obtained by variation with respect to, say, $\Delta e_{a \bar{b}}$. In total, the variation of (3.1) can be written as

$$
\delta S=\int d x d \tilde{x} e^{-2 d}\left(-2 \delta d \mathcal{R}+\Delta e_{a \bar{b}} \mathcal{R}^{a \bar{b}}\right)
$$

giving rise to the field equations

$$
\mathcal{R}=0, \quad \mathcal{R}_{a \bar{b}}=0 .
$$

Next we discuss some general properties of these tensors. As indicated by the suggestive notation it is natural to assume that the 'Ricci tensor' $\mathcal{R}_{a \bar{b}}$ derived from (3.1) indeed follows from contracting

the covariant curvature tensor introduced above. There are two candidates, $\mathcal{R}_{\bar{c} a \bar{b}}{ }^{\bar{c}}$ and $\mathcal{R}_{c \bar{b} a}{ }^{c}$. The explicit expression for the first is

$$
\begin{aligned}
\mathcal{R}_{a \bar{b}}=2 \mathcal{R}_{\bar{c} a \bar{b}}^{\bar{c}}=R_{\bar{c} a \bar{b}}^{\bar{c}} & =e_{\bar{c}} \omega_{a \bar{b}}^{\bar{c}}-e_{a} \omega_{\bar{c} \bar{b}}^{\bar{c}}+\omega_{\bar{c} \bar{b}}{ }^{\bar{d}} \omega_{a \bar{d}}{ }^{\bar{c}}-\omega_{a \bar{b}}{ }^{\bar{d}} \omega_{\bar{c} \bar{d}}{ }^{\bar{c}}-\Omega_{\bar{c} a}{ }^{E} \omega_{E \bar{b}} \bar{c} \\
& =e_{\bar{c}} \omega_{a \bar{b}}{ }^{\bar{c}}-e_{a} \omega_{\bar{c} \bar{b}}{ }^{\bar{c}}+\omega_{d \bar{b}}{ }^{\bar{c}} \omega_{\bar{c} a}{ }^{d}-\omega_{a \bar{b}} \omega_{\bar{c} \bar{d}}{ }^{\bar{c}}
\end{aligned}
$$

where the torsion constraint (2.26) has been used in the first line. The second expression is given by

$$
\begin{aligned}
\mathcal{R}_{\bar{b} a}=R_{c \bar{b} a}{ }^{c} & =e_{c} \omega_{\bar{b} a}{ }^{c}-e_{\bar{b}} \omega_{c a}{ }^{c}+\omega_{c a}{ }^{d} \omega_{\bar{b} d}{ }^{c}-\omega_{\bar{b} a}{ }^{d} \omega_{c d}^{c}-\Omega_{c \bar{b}}{ }^{E} \omega_{E a}{ }^{c} \\
& =e_{c} \omega_{\bar{b} a}{ }^{c}-e_{\bar{b}} \omega_{c a}{ }^{c}+\omega_{\bar{d} a}{ }^{c} \omega_{c \bar{b}}{ }^{\bar{d}}-\omega_{\bar{b} a}{ }^{d} \omega_{c d}{ }^{c}
\end{aligned}
$$


and we will confirm below that this is equivalent to (3.7). Writing out all connection components explicitly, the Ricci tensor can thus be written as

$$
\mathcal{R}_{a \bar{b}}=\mathcal{R}_{\bar{b} a}=e_{\bar{b}} \tilde{\Omega}_{a}-e_{c} \Omega_{\bar{b} a}{ }^{c}+\Omega_{c \bar{b}}{ }^{\bar{d}} \Omega_{\bar{d} a}{ }^{c}-\Omega_{\bar{b} a}{ }^{c} \tilde{\Omega}_{c} .
$$

In sec. 4 we will prove that the curvature scalar, upon gauge fixing, reduces to the one of double field theory given in [8], and that the corresponding field equations for $\mathcal{E}_{i j}$ as determined in [11] give rise to the tensors in (3.7) or (3.8), thus showing their equivalence. This proves that the tensors defined by the general variation (3.5) are indeed the curvature scalar and Ricci tensor.

\subsection{Covariant gauge variation and Bianchi identity}

In this subsection we derive a Bianchi identity from the invariance of (3.1) under $\xi^{M}$ gauge transformations. To this end it is convenient to first rewrite the gauge transformations in terms of the $G L(D) \times G L(D)$ covariant derivatives. For this we use the following form of the gauge transformation in terms of the C-bracket (c.f. eqs. (3.29) and (3.30) in [9])

$$
\delta_{\xi} e_{A}^{M}=\left[\xi, e_{A}\right]_{\mathrm{C}}^{M}+\frac{1}{2} \partial^{M}\left(e_{A}^{N} \xi_{N}\right)
$$

and the fact that in the $\mathrm{C}$ bracket we can replace curved by flat indices if we use the $G L(D) \times G L(D)$ covariant derivatives, i.e.,

$$
\begin{aligned}
{\left[\xi, e_{A}\right]_{\mathrm{C}}^{B} } & =\xi^{C} \hat{\nabla}_{C} e_{A}{ }^{B}-e_{A}{ }^{C} \nabla_{C} \xi^{B}-\frac{1}{2} \xi_{C} \hat{\nabla}^{B} e_{A}{ }^{C}+\frac{1}{2} e_{A C} \nabla^{B} \xi^{C} \\
& =-\xi^{C} \omega_{C A}{ }^{B}-\nabla_{A} \xi^{B}+\frac{1}{2} \xi^{C} \omega^{B}{ }_{A C}+\frac{1}{2} \mathcal{G}_{A C} \nabla^{B} \xi^{C} .
\end{aligned}
$$

Here we have to stress that the covariant derivatives in the first line do not act on the index $A$, which we indicated by the notation $\hat{\nabla}$, because $A$ is in (3.10) and (3.11) only a 'spectator' index. Consequently, using $e_{A}{ }^{B} \equiv e_{A}{ }^{M} e_{M}{ }^{B}=\delta_{A}{ }^{B}$ and $e_{A C} \equiv e_{A M} e_{C}{ }^{M}=\mathcal{G}_{A C}$, we have $\hat{\nabla}_{C} e_{A}{ }^{B}=-\omega_{C A}{ }^{B}$, from which the second equality follows. Using (3.11) in (3.10) we obtain

$$
\begin{aligned}
\delta_{\xi} e_{A}{ }^{M} & =e_{B}{ }^{M}\left[\xi, e_{A}\right]_{\mathrm{C}}^{B}+\frac{1}{2} \partial^{M} \xi_{A} \\
& =-\xi^{C} \omega_{C A}{ }^{B} e_{B}{ }^{M}-e_{B}{ }^{M} \nabla_{A} \xi^{B}+\frac{1}{2} e_{B}{ }^{M} \omega^{B}{ }_{A C} \xi^{C}+\frac{1}{2} e_{B}{ }^{M} \nabla^{B} \xi_{A}+\frac{1}{2} \partial^{M} \xi_{A} .
\end{aligned}
$$

The third and last term combine into a covariant derivative, which in turn combines with the fourth term. Moreover, the first term can be viewed as a field-dependent $G L(D) \times G L(D)$ transformation with parameter $\Lambda_{A}^{B}=-\xi^{C} \omega_{C A}{ }^{B}$ and can thus be discarded. Therefore, the final form reads

$$
\delta_{\xi} e_{A}^{M}=-e_{B}^{M}\left(\nabla_{A} \xi^{B}-\nabla^{B} \xi_{A}\right),
$$

or, in terms of the variation (3.2),

$$
\Delta e_{A B}=\nabla_{B} \xi_{A}-\nabla_{A} \xi_{B}
$$


For the dilaton one finds from (1.6)

$$
\begin{aligned}
\delta_{\xi} d & =\xi^{M} \partial_{M} d-\frac{1}{2} \partial_{M} \xi^{M}=\xi^{A} e_{A} d-\frac{1}{2} \partial_{M}\left(\xi^{A} e_{A}^{M}\right) \\
& =-\frac{1}{2} e_{A} \xi^{A}+\frac{1}{2} \xi^{A}\left(-\partial_{M} e_{A}^{M}+2 e_{A} d\right)=-\frac{1}{2}\left(e_{A} \xi^{A}-\omega_{B A}{ }^{B} \xi^{A}\right) \\
& =-\frac{1}{2} \nabla_{A} \xi^{A}
\end{aligned}
$$

where we used (2.37) in the second line.

We can now read off the Bianchi identity following from the gauge invariance of (3.1). Using (3.14) and (3.15) in (3.5) we infer

$$
\begin{aligned}
0 & =\delta_{\xi} S=\int d x d \tilde{x} e^{-2 d}\left(\left(\nabla_{a} \xi^{a}+\nabla_{\bar{a}} \xi^{\bar{a}}\right) \mathcal{R}+\left(\nabla_{\bar{b}} \xi_{a}-\nabla_{a} \xi_{\bar{b}}\right) \mathcal{R}^{a \bar{b}}\right) \\
& =-\int d x d \tilde{x} e^{-2 d}\left(\xi^{a}\left(\nabla_{a} \mathcal{R}+\nabla^{\bar{b}} \mathcal{R}_{a \bar{b}}\right)+\xi^{\bar{a}}\left(\nabla_{\bar{a}} \mathcal{R}-\nabla^{b} \mathcal{R}_{b \bar{a}}\right)\right)
\end{aligned}
$$

which implies the Bianchi identities [16]

$$
\nabla_{a} \mathcal{R}+\nabla^{\bar{b}} \mathcal{R}_{a \bar{b}}=0, \quad \nabla_{\bar{a}} \mathcal{R}-\nabla^{b} \mathcal{R}_{b \bar{a}}=0
$$

These are equivalent to similar Bianchi identities derived from the double field theory, as we will show in the next section, and reduce to the usual Bianchi identities for $R_{i j}$ and $H_{i j k}$ when $\tilde{\partial}=0$ [11].

\section{Relation to formulation with $\mathcal{E}_{i j}$}

Here we start the detailed 're-derivation' of the double field theory formulations reviewed in the introduction from Siegel's geometrical formalism. We identify the 'non-symmetric' metric $\mathcal{E}_{i j}$ as components of $e_{A}^{M}$ after a particular gauge fixing. This allows us to study the non-linear realization of the $O(D, D)$ symmetry and to find a rather direct relation between the action (1.2) and the geometrical Einstein-Hilbert like action (3.1).

\subsection{Gauge choice}

One way to identify $\mathcal{E}_{i j}$ in the frame-like formalism is to gauge-fix the local $G L(D) \times G L(D)$ symmetry by setting the components $e_{a}^{i}$ and $e_{\bar{a}}{ }^{i}$ in (2.10) equal to the unit matrix (assuming certain invertibility properties). Taking the constraint (2.13) into account, the remaining components are then parametrized by a general $D \times D$ matrix which we identify with $\mathcal{E}_{i j}, 7$

$$
e_{A}^{M}=\left(\begin{array}{cc}
e_{a i} & e_{a}{ }^{i} \\
e_{\bar{a} i} & e_{\bar{a}}{ }^{i}
\end{array}\right)=\left(\begin{array}{cc}
-\mathcal{E}_{a i} & \delta_{a}{ }^{i} \\
\mathcal{E}_{i \bar{a}} & \delta_{\bar{a}}{ }^{i}
\end{array}\right)
$$

\footnotetext{
${ }^{7}$ An alternative definition of $\mathcal{E}_{i j}$ in terms of the frame fields which is $G L(D) \times G L(D)$ covariant and does not require a gauge fixing has been given in 9 . For our present purposes, however, we find it more convenient to use the gauge fixed form (4.1).
} 
In this gauge, the 'space-time' indices $i, j, \ldots$ can be identified with the frame indices of either $G L(D)$ factor via the trivial vielbeins $\delta_{a}{ }^{i}$ or $\delta_{\bar{a}}{ }^{i}$. The calligraphic derivatives (1.3) then coincide with the 'flattened' partial derivatives (2.14),

$$
e_{a}=e_{a}{ }^{M} \partial_{M}=\partial_{a}-\mathcal{E}_{a i} \tilde{\partial}^{i} \equiv \mathcal{D}_{a}, \quad e_{\bar{a}}=e_{\bar{a}}{ }^{M} \partial_{M}=\partial_{\bar{a}}+\mathcal{E}_{i \bar{a}} \tilde{\partial}^{i} \equiv \overline{\mathcal{D}}_{\bar{a}}
$$

Moreover, the metric $g_{i j}=\mathcal{E}_{(i j)}$ can be identified with either of the two 'tangent space' metrics

$$
g_{a b}=-\frac{1}{2} e_{a}^{M} e_{b}{ }^{N} \eta_{M N}, \quad g_{\bar{a} \bar{b}}=\frac{1}{2} e_{\bar{a}}^{M} e_{\bar{b}}{ }^{N} \eta_{M N}
$$

as one may verify directly from (4.1). From this it follows that (2.11) is given by

$$
\mathcal{G}_{A B}=\left(\begin{array}{cc}
-2 g_{a b} & 0 \\
0 & 2 g_{\bar{a} \bar{b}}
\end{array}\right)
$$

The relative factors of \pm 2 appearing here lead, after the gauge fixing (4.1) and the corresponding identification of indices, to an ambiguity regarding the contraction of indices. We will follow the convention that contractions are done with respect to the tangent space metric $\mathcal{G}_{A B}$ when the indices are letters from the beginning of the latin alphabet (i.e., either $a, b \ldots$ or $\bar{a}, \bar{b}, \ldots$ ), and that contractions are only done with respect to $g_{i j}$ if the indices are letters from the middle of the latin alphabet $(i, j, \ldots)$.

For the comparison with the action (1.2) it is instructive to re-interpret derivatives like $\mathcal{D}_{i} \mathcal{E}_{j k}$ in a more covariant way. Specifically, in analogy to the modified variation (3.2), we can write this as

$$
\mathcal{D}_{a} \mathcal{E}_{b \bar{c}}=e_{b}{ }^{M} e_{a} e_{\bar{c} M}=-e_{\bar{c}}^{M} e_{a} e_{b M} .
$$

This follows from the gauge fixed forms (4.1) and (4.2), and is manifestly $O(D, D)$ invariant. Remarkably, it can also be made manifestly $G L(D) \times G L(D)$ invariant by observing that in

$$
e_{b}{ }^{M} \nabla_{a} e_{\bar{c} M}=e_{b}{ }^{M}\left(e_{a} e_{\bar{c} M}+\omega_{a \bar{c}}{ }^{\bar{d}} e_{\bar{d} M}\right)
$$

the connection term is zero by the constraint (2.13). The same conclusion applies to the barred derivative $e_{\bar{a}}=\overline{\mathcal{D}}_{\bar{a}}$, and so we find in total the following identifications

$$
\begin{aligned}
& \mathcal{D}_{a} \mathcal{E}_{b \bar{c}} \equiv e_{b}{ }^{M} \nabla_{a} e_{\bar{c} M}=-e_{\bar{c}}{ }^{M} \nabla_{a} e_{b M}, \\
& \overline{\mathcal{D}}_{\bar{a}} \mathcal{E}_{b \bar{c}} \equiv e_{b}{ }^{M} \nabla_{\bar{a}} e_{\bar{c} M}=-e_{\bar{c}}{ }^{M} \nabla_{\bar{a}} e_{b M},
\end{aligned}
$$

which are manifestly covariant with respect to $O(D, D)$ and tangent space transformations.

In the following we will examine how the $O(D, D)$ duality symmetry is realized after this gauge fixing. Acting with a general $O(D, D)$ transformation on (4.1) violates the gauge condition and thus requires a compensating $G L(D) \times G L(D)$ transformation. In order to determine the transformation that restores the form of the vielbein (4.1), we consider a finite $O(D, D)$ and $G L(D)$ transformation,

$$
e_{a}^{M \prime}\left(X^{\prime}\right)=h^{M}{ }_{N}\left(M^{-1}(X)\right)_{a}{ }^{b} e_{b}{ }^{N}(X) .
$$

Here we denoted the $G L(D)$ matrix by $M^{-1}$ for later convenience, and $h$ is the $O(D, D)$ matrix in (1.5), i.e., whose components read

$$
h_{N}^{M}=\left(\begin{array}{ll}
h_{i}{ }^{j} & h_{i j} \\
h^{i j} & h^{i}{ }_{j}
\end{array}\right)=\left(\begin{array}{ll}
a_{i}{ }^{j} & b_{i j} \\
c^{i j} & d^{i}{ }_{j}
\end{array}\right) .
$$


Applied to the gauge fixed component we find

$$
e_{a}^{i \prime}=\left(M^{-1}\right)_{a}{ }^{b}\left(h^{i}{ }_{j} e_{b}^{j}+h^{i j} e_{b j}\right)=\left(M^{-1}\left(d^{t}-\mathcal{E} c^{t}\right)\right)_{a}{ }^{i}=\delta_{a}{ }^{i},
$$

where we used matrix notation and suppressed the $X$-dependence. The last equation expresses the condition that the gauge fixing condition be preserved. Analogously, one finds for the other component

$$
e_{\bar{a}}^{i \prime}=\left(\bar{M}^{-1}\right)_{\bar{a}}{ }^{\bar{b}}\left(h^{i}{ }_{j} e_{\bar{b}}^{j}+h^{i j} e_{\bar{b} j}\right)=\left(\bar{M}^{-1}\left(d^{t}+\mathcal{E}^{t} c^{t}\right)\right)_{\bar{a}}^{i}=\delta_{\bar{a}}{ }^{i}
$$

where we denoted the matrix corresponding to the second $G L(D)$ factor by $\bar{M}^{-1}$. The two conditions (4.10) and (4.11) thus determine the compensating $G L(D) \times G L(D)$ transformations uniquely in terms of $c$ and $d$,

$$
M(X)=d^{t}-\mathcal{E}(X) c^{t}, \quad \bar{M}(X)=d^{t}+\mathcal{E}^{t}(X) c^{t},
$$

which are both $X$-dependent through their dependence on $\mathcal{E}_{i j}$. Finally, using this form of the compensating gauge transformations it is straightforward to verify that $\mathcal{E}_{i j}$ transforms under $O(D, D)$ in the required non-linear representation according to (1.4).

With the above analysis of the non-linear realization of $O(D, D)$ we have in fact recovered the formalism that has been used in [8] (extending the background-dependent formalism in [21, 6]) in order to prove the $O(D, D)$ invariance of (1.2). More precisely, in this formalism every index is thought of either as an unbarred or barred index and to transform, accordingly, either under $M$ or $\bar{M}$ in (4.12). For instance, we have just verified that the calligraphic derivatives (4.2) transform with $M$ or $\bar{M}$, respectively. Moreover, due to the manifestly $O(D, D)$ and $G L(D) \times G L(D)$ covariant rewriting of the calligraphic derivatives of $\mathcal{E}$ in (4.7), it follows that after gauge fixing

$$
\mathcal{D}_{a} \mathcal{E}_{b \bar{c}}=M_{a}{ }^{d} M_{b}{ }^{e} \bar{M}_{\bar{c}}^{\bar{f}} \mathcal{D}_{d}^{\prime} \mathcal{E}_{e \bar{f}}^{\prime}, \quad \overline{\mathcal{D}}_{\bar{a}} \mathcal{E}_{b \bar{c}}=\bar{M}_{\bar{a}}^{\bar{d}} M_{b}{ }^{e} \bar{M}_{\bar{c}}^{\bar{f}} \overline{\mathcal{D}}_{\bar{d}}^{\prime} \mathcal{E}_{e \bar{f}}^{\prime}
$$

Thus, we can think of the first index on $\mathcal{E}$ (under $\mathcal{D}$ or $\overline{\mathcal{D}}$ ) as unbarred and the second index as barred. From the definition (4.3) we infer that the indices on $g$ can be thought of either as both barred or both unbarred, because $g$ can be viewed as a tensor either of the left $G L(D)$ or the right $G L(D)$ such that it transforms after gauge fixing as

$$
g_{\bar{a} \bar{b}}=\bar{M}_{\bar{a}}^{\bar{c}} \bar{M}_{\bar{b}}^{\bar{d}} g_{\bar{c} \bar{d}}^{\prime}, \quad g_{a b}=M_{a}^{c} M_{b}^{d} g_{c d}^{\prime},
$$

and similarly for the inverse. The $O(D, D)$ invariance of the action is then a consequence of the fact, which one may easily confirm by inspection of (1.2), that only like-wise indices are contracted [8].

\section{2 $O(D, D)$ covariant derivatives and gauge variation}

In the previous subsection we have seen that in the formulation using $\mathcal{E}_{i j}$ the $O(D, D)$ transformations are governed by the matrices $M$ and $\bar{M}$ in (4.12). Since these matrices are $X$-dependent, it follows that derivatives of objects that transform 'covariantly' with $M$ and $\bar{M}$ according to their index structure are in general not covariant in the same sense. This led ref. [8] to introduce ' $O(D, D)$ covariant derivatives' - despite $O(D, D)$ being a global symmetry with constant parameters. There are two 
types of covariant derivatives, $\nabla_{i}(\Gamma)$ and $\bar{\nabla}_{i}(\Gamma)$, i.e., unbarred and barred, and various connections $\Gamma$ depending on the index structure of the object on which the derivative acts. Here we indicate the dependence on the connections explicitly, in order to distinguish these 'covariant' derivatives from the $G L(D) \times G L(D)$ covariant derivatives introduced before.

Since we have here realized the global non-linear $O(D, D)$ transformations according to $M$ and $\bar{M}$ through compensating $G L(D) \times G L(D)$ transformations, it is natural to assume that, after gauge fixing, the $G L(D) \times G L(D)$ covariant derivatives are related to the ' $O(D, D)$ covariant derivatives' of [8. This indeed turns out to be the case, and so we are able to give a more conventional interpretation of these covariant derivatives.

As a first test of this relation we reproduce a manifestly $O(D, D)$ covariant form of the $\xi^{M}$ gauge transformations that has been found in [8]. Specifically, introducing the following change of basis for the gauge parameters (which is suggested by the gauge structure in string field theory [6]),

$$
\eta_{i}=-\tilde{\xi}_{i}+\mathcal{E}_{i j} \xi^{j}, \quad \bar{\eta}_{i}=\tilde{\xi}_{i}+\xi^{j} \mathcal{E}_{j i},
$$

the gauge transformations (1.6) take the remarkable form

$$
\delta \mathcal{E}_{i j}=\nabla_{i}(\Gamma) \bar{\eta}_{j}+\bar{\nabla}_{j}(\Gamma) \eta_{i}
$$

The corresponding result using the $G L(D) \times G L(D)$ connections follows almost immediately. First, the flattened gauge parameters

$$
\eta_{a}:=-\xi_{a} \equiv-e_{a}^{M} \xi_{M}, \quad \bar{\eta}_{\bar{a}}:=\xi_{\bar{a}} \equiv e_{\bar{a}}{ }^{M} \xi_{M},
$$

coincide with (4.15) upon using (4.1). Moreover, after the gauge fixing (4.1), any variation of $\mathcal{E}$ coincides with the $\Delta$ variation in (3.2),

$$
\delta \mathcal{E}_{a \bar{b}}=\Delta e_{\bar{b} a}=e_{a}^{M} \delta e_{\bar{b} M}=e_{a}^{i} \delta e_{\bar{b} i}+e_{a i} \delta e_{\bar{b}}^{i} .
$$

This follows because the last term is zero by the gauge fixing condition. More precisely, for the $\xi^{M}$ gauge variation this term will vanish by a compensating frame rotation that restores the chosen gauge. The advantage of using the $\Delta$ variation is that this compensating transformation need not be determined explicitly. Applying now (3.14) one finds in the basis (4.17)

$$
\delta \mathcal{E}_{a \bar{b}}=\nabla_{a} \bar{\eta}_{\bar{b}}+\nabla_{\bar{b}} \eta_{a},
$$

which agrees with (4.16), using that after gauge fixing the indices $i, j, \ldots$ can be identified with the flat indices.

We note in passing that the original form (1.6) of the gauge transformations also follows easily by use of the $\Delta$ variation as in (4.18),

$$
\begin{aligned}
\delta \mathcal{E}_{a \bar{b}} & =\Delta e_{\bar{b} a}=e_{a}{ }^{M} \delta e_{\bar{b} M}=e_{a}{ }^{M}\left(\xi^{N} \partial_{N} e_{\bar{b} M}+\left(\partial_{M} \xi^{N}-\partial^{N} \xi_{M}\right) e_{\bar{b} N}\right) \\
& =\xi^{N} \partial_{N} \mathcal{E}_{a \bar{b}}+\mathcal{D}_{a} \xi^{N} e_{\bar{b} N}-\overline{\mathcal{D}}_{\bar{b}} \xi_{M} e_{a}{ }^{M} \\
& =\xi^{N} \partial_{N} \mathcal{E}_{a \bar{b}}+\mathcal{D}_{a} \tilde{\xi}_{j} e_{\bar{b}}^{j}+\mathcal{D}_{a} \xi^{j} e_{\bar{b} j}-\overline{\mathcal{D}}_{\bar{b}} \tilde{\xi}_{j} e_{a}^{j}-\overline{\mathcal{D}}_{\bar{b}} \xi^{j} e_{a j} \\
& =\xi^{N} \partial_{N} \mathcal{E}_{a \bar{b}}+\mathcal{D}_{a} \tilde{\xi}_{\bar{b}}+\mathcal{D}_{a} \xi^{j} \mathcal{E}_{j \bar{b}}-\overline{\mathcal{D}}_{\bar{b}} \tilde{\xi}_{a}+\overline{\mathcal{D}}_{\bar{b}} \xi^{j} \mathcal{E}_{a j} .
\end{aligned}
$$


Here we used (4.2) in the second line and the gauge fixed form (4.1) in the last line, where we again identified indices. Thus we have derived (1.6) from the fundamental gauge transformation of the vielbein, as in [9], but without invoking the compensating frame rotation explicitly.

The previous results show that the ' $O(D, D)$ covariant derivatives' coincide with the $G L(D) \times$ $G L(D)$ covariant derivatives after gauge fixing, at least when acting on $\eta$ and $\bar{\eta}$ as in (4.19). The complete set of connections $\Gamma$ is not fixed by $O(D, D)$ covariance and therefore have been given in 8 only provisionally. Here we display for completeness their relation after gauge fixing,

$$
\begin{aligned}
\omega_{i \bar{j}}{ }^{\bar{k}} & =-\frac{1}{2} g^{k l}\left(\mathcal{D}_{i} \mathcal{E}_{l j}+\overline{\mathcal{D}}_{j} \mathcal{E}_{i l}-\overline{\mathcal{D}}_{l} \mathcal{E}_{i j}\right)=-\Gamma_{i \bar{j}}^{\bar{k}}, \\
\omega_{i j}{ }^{k} & =-\frac{1}{2} g^{k l}\left(\overline{\mathcal{D}}_{i} \mathcal{E}_{j l}+\mathcal{D}_{j} \mathcal{E}_{l i}-\mathcal{D}_{l} \mathcal{E}_{j i}\right)=-\Gamma_{\overline{i j}}^{k}, \\
\omega_{j i}{ }^{j} & =\frac{1}{2}\left(\overline{\mathcal{D}}^{j}-\mathcal{D}^{j}\right) \mathcal{E}_{i j}+2 \mathcal{D}_{i} d=-\Gamma_{j i}^{j}+\frac{1}{2} \overline{\mathcal{D}}^{j} \mathcal{E}_{i j}+2 \mathcal{D}_{i} d, \\
\omega_{\overline{j i}} \bar{j} & =\frac{1}{2}\left(\mathcal{D}^{j}-\overline{\mathcal{D}}^{j}\right) \mathcal{E}_{j i}+2 \overline{\mathcal{D}}_{i} d=-\Gamma_{\overline{j i}}^{\bar{j}}+\frac{1}{2} \mathcal{D}^{j} \mathcal{E}_{j i}+2 \overline{\mathcal{D}}_{i} d .
\end{aligned}
$$

We see that they are equivalent in the 'off-diagonal' parts but differ in the trace parts. In fact, it has already been noted, c.f. the discussion around eq. (4.13) in [8], that modifying the definition as suggested by (4.21) would have the advantage of simplifying the gauge transformation of $d$ in that

$$
\delta d=-\frac{1}{4} \nabla_{i} \eta^{i}-\frac{1}{4} \bar{\nabla}_{i} \bar{\eta}^{i}
$$

Here we see that this is a direct consequence of (3.15), where we recall that according to our index conventions $g$ rather than $\mathcal{G}$ is used to raise indices in (4.22), and that there is a relative sign in the definition (4.17) of $\eta_{i}$. In [8], however, there was no justification from symmetry arguments for this modification, but here we see it emerging naturally from Siegel's frame formalism.

Given the precise correspondence between the $O(D, D)$ and $G L(D) \times G L(D)$ connections, we have verified that the curvature scalar and Ricci tensor of Siegel's formalism agree with the corresponding expressions obtained in [8] and [11] (for the Ricci tensor see appendix A4). More precisely, the scalar curvature constructed from Siegel's frame formalism is $\frac{1}{4}$ times $\mathcal{R}(\mathcal{E}, d)$ as given in [8]. Taking this factor as well as the relative factors of $\pm \frac{1}{2}$ in (4.3) into account, the Bianchi identities (3.17) reduce to

$$
\nabla^{i} \mathcal{R}_{i j}+\frac{1}{2} \overline{\mathcal{D}}_{j} \mathcal{R}(\mathcal{E}, d)=0, \quad \bar{\nabla}^{j} \mathcal{R}_{i j}+\frac{1}{2} \mathcal{D}_{i} \mathcal{R}(\mathcal{E}, d)=0,
$$

which agree with [1].

Starting from the expression (2.59) for the scalar curvature we can actually immediately compare with the double field theory action (1.2) in terms of $\mathcal{E}_{i j}$. Using that the covariant derivatives allow for partial integration in presence of the dilaton density, we infer that the first line in (2.59) contributes only total derivatives under an integral, and thus the resulting Lagrangian is equivalent to

$$
\begin{aligned}
\mathcal{L}^{\prime}=e^{-2 d} & -\frac{1}{2} e^{a M} \nabla^{b} e^{\bar{c}}{ }_{M} e_{a}{ }^{N} \nabla_{b} e_{\bar{c} N}+\frac{1}{2} e_{\bar{c}}{ }^{M} \nabla_{a} e_{b M} e^{\bar{c} N} \nabla^{b} e^{a}{ }_{N}-\frac{1}{2} e_{c}{ }^{M} \bar{\nabla}_{\bar{a}} e_{\bar{b} M} e^{c N} \bar{\nabla}^{\bar{b}} e^{\bar{a}}{ }_{N} \\
& \left.-\nabla^{a} d e_{a}{ }^{M} \bar{\nabla}^{\bar{b}} e_{\bar{b} M}+\bar{\nabla}^{\bar{a}} d e_{\bar{a}}{ }^{M} \nabla^{b} e_{b M}-2 \nabla^{a} d \nabla_{a} d\right) .
\end{aligned}
$$

Taking into account the relation (4.3) between $g$ and the tangent space metric, and using that the latter is covariantly constant, it then immediately follows by virtue of the identifications (4.7) that this agrees with (1.2) up to the overall factor of 4 . 


\section{$5 \quad$ Relation to formulation with $\mathcal{H}^{M N}$}

In this section we introduce the formulation in terms of the generalized metric $\mathcal{H}^{M N}$ from the point of view of the frame formalism and express the scalar curvature and thus the action in terms of this variable. Finally, we briefly discuss Christoffel connections that are introduced via a vielbein postulate.

\subsection{Gauge choice and generalized coset formulation}

We next identify the generalized metric and the corresponding formulation (1.9) in the geometrical frame formalism. In general, one can define $\mathcal{H}^{M N}$ in terms of the frame field through [9]

$$
\mathcal{H}^{M N}=2 \mathcal{G}^{\bar{a} \bar{b}} e_{\bar{a}}{ }^{M} e_{\bar{b}}{ }^{N}-\eta^{M N}=-2 \mathcal{G}^{a b} e_{a}{ }^{M} e_{b}{ }^{N}+\eta^{M N},
$$

where the second equation is a consequence of the definition (2.11) and the constraint (2.13). The generalized metric is a constrained field in that

$$
\mathcal{H}^{M K} \mathcal{H}_{K N}=\delta^{M}{ }_{N}
$$

where the indices are lowered, as usual, with $\eta_{M N}$. In the standard parametrization (1.8) this can be checked by a direct computation. Here, it can be verified with either one of the definitions in (5.1). We note, however, that if we use for the first $\mathcal{H}$ in (5.2), say, the first expression in (5.1) and for the second $\mathcal{H}$ the second expression, then the constraint $(2.13)$ is required in order to verify this.

For later use we note that (5.1) implies for the flattened components of the generalized metric

$$
\mathcal{H}^{A B}=\mathcal{H}^{M N} e_{M}{ }^{A} e_{N}{ }^{B}=\left(\begin{array}{cc}
-\mathcal{G}^{a b} & 0 \\
0 & \mathcal{G}^{\bar{a} \bar{b}}
\end{array}\right)
$$

where again (2.13) has been used.

In the following, we find it convenient to fix the $G L(D) \times G L(D)$ symmetry by setting the tangent space metric (2.11) to

$$
\mathcal{G}_{A B}=\left(\begin{array}{cc}
-\delta_{a b} & 0 \\
0 & \delta_{\bar{a} \bar{b}}
\end{array}\right)
$$

This implies $g_{a b}=\frac{1}{2} \delta_{a b}$ and $g_{\bar{a} \bar{b}}=\frac{1}{2} \delta_{\bar{a} \bar{b}}$ from the definition (4.3) and also $\mathcal{H}_{A B}=\delta_{A B}$ from (5.3). This leaves a residual local $O(D) \times O(D)$ symmetry. Therefore, the resulting formulation can be viewed as a generalized coset model based on $O(D, D) /(O(D) \times O(D))$ [9]. In fact, from (5.4) we conclude with (2.11) that $e_{A}^{M}$ is an $O(D, D)$ element (up to a similarity transformation) in that it transforms the $O(D, D)$ metric $\eta$ into the $O(D, D)$ metric, but written in the form (5.4). Thus, $e$ can be viewed as a group-valued coset representative with a local $O(D) \times O(D)$ action from the left. Moreover, (5.3) implies

$$
\mathcal{H}^{M N}=\delta^{A B} e_{A}^{M} e_{B}^{N},
$$

and so $\mathcal{H}$ can be viewed as the $O(D) \times O(D)$ invariant combination $e^{t} e$. For completeness we record that the form of the coset representative that leads to the standard parametrization (1.8) for $\mathcal{H}^{M N}$ according to (5.5) is given by

$$
e_{A}^{M}=\frac{1}{\sqrt{2}}\left(\begin{array}{cc}
v_{a i}+b_{i j} v_{a}^{j} & v_{a}^{i} \\
-v_{\bar{a} i}+b_{i j} v_{\bar{a}}^{j} & v_{\bar{a}}^{i}
\end{array}\right)
$$


where $v_{i}{ }^{a}$ is the conventional vielbein for the metric $g_{i j}$, i.e., $g_{i j}=v_{i}{ }^{a} v_{j a}$, with inverse $v_{a}^{i}$. We recall that an explicit parametrization like this requires a further gauge fixing of the local $O(D) \times O(D)$ symmetry.

\subsection{Scalar curvature}

Next, we prove that the Ricci scalar (2.60) reduces upon the gauge fixing (5.4) to the function $\mathcal{R}(\mathcal{H}, d)$ given in [9], and thus that the actions in (3.1) and (1.11) are equivalent. This proof simplifies due to the fact that we have chosen a gauge in which $\mathcal{G}_{A B}$ is constant, such that we can freely raise and lower indices $a, b, \ldots$ and $\bar{a}, \bar{b}, \ldots$ under derivatives. Thus, it implies relations like

$$
f_{A B C}=\left(e_{A} e_{B}^{M}\right) e_{C M}=-e_{B}^{M}\left(e_{A} e_{C M}\right)=-f_{A C B},
$$

which we will use frequently below. Moreover, the scalar curvature (2.60) then reduces to

$$
\mathcal{R}=e_{a} \tilde{\Omega}^{a}+\frac{1}{2} \tilde{\Omega}_{a}^{2}-\frac{1}{4} \Omega_{a b \bar{c}}^{2}-\frac{1}{12} \Omega_{[a b c]}^{2} .
$$

We first evaluate the dilaton-dependent terms, which originate only from the first two terms. Using (2.37) we find

$$
\begin{aligned}
e_{a} \tilde{\Omega}^{a}+\left.\frac{1}{2} \tilde{\Omega}_{a}^{2}\right|_{d} & =-2 e^{a N} \partial_{N}\left(e_{a}^{M} \partial_{M} d\right)-2 \partial_{M} e_{a}^{M} e^{a N} \partial_{N} d+2 e_{a}{ }^{M} \partial_{M} d e^{a N} \partial_{N} d \\
& =-2 e^{a N} e_{a}{ }^{M} \partial_{M} \partial_{N} d-2 \partial_{N}\left(e^{a N} e_{a}{ }^{M}\right) \partial_{M} d+2 e_{a}{ }^{M} e^{a N} \partial_{M} d \partial_{N} d
\end{aligned}
$$

With the expression for $\mathcal{H}^{M N}$ from (5.1) this reduces to

$$
e_{a} \tilde{\Omega}^{a}+\left.\frac{1}{2} \tilde{\Omega}_{a}^{2}\right|_{d}=\mathcal{H}^{M N} \partial_{M} \partial_{N} d+\partial_{N} \mathcal{H}^{M N} \partial_{M} d-\mathcal{H}^{M N} \partial_{M} d \partial_{N} d
$$

where we used the strong constraint (1.7).

We turn next to the pure $e$-dependent terms which are more involved. The first two terms in (5.8) yield

$$
e_{a} \tilde{\Omega}^{a}+\left.\frac{1}{2} \tilde{\Omega}_{a}^{2}\right|_{e}=\frac{1}{2}\left(\partial_{M} \partial_{N}\left(e_{a}^{N} e^{a M}\right)-\partial_{N} e^{a M} \partial_{M} e_{a}^{N}\right)=-\frac{1}{4} \partial_{M} \partial_{N} \mathcal{H}^{M N}-\frac{1}{2} \partial_{N} e^{a M} \partial_{M} e_{a}{ }^{N} .
$$

In order to compute the third term in (5.8) we start from

$$
\Omega_{a b \bar{c}}=2 f_{[a b] \bar{c}}+f_{\bar{c}[a b]}=2\left(e_{[a} e_{b]}^{M}\right) e_{\bar{c} M}+\left(e_{\bar{c}} e_{a}{ }^{M}\right) e_{b M},
$$

where (5.7) implies automatic antisymmetry in $a, b$ in the last term. Using that for arbitrary functions $X$ and $Y$ the strong constraint (2.15) implies together with (5.1)

$$
e_{a} X e^{a} Y=-\frac{1}{2} \mathcal{H}^{M N} \partial_{M} X \partial_{N} Y, \quad e_{\bar{a}} X e^{\bar{a}} Y=\frac{1}{2} \mathcal{H}^{M N} \partial_{M} X \partial_{N} X
$$

the square of (5.12) reads

$$
\begin{aligned}
\Omega_{a b \bar{c}}{ }^{2}= & -\frac{1}{2} \mathcal{H}^{K L}\left(\mathcal{H}_{M N}+\eta_{M N}\right) \partial_{K} e_{b}{ }^{M} \partial_{L} e^{b N}-\left(\mathcal{H}_{M N}+\eta_{M N}\right) e_{b} e_{a}{ }^{M} e^{a} e^{b N} \\
& -\frac{1}{4} \mathcal{H}^{K L}\left(\mathcal{H}_{M N}-\eta_{M N}\right) \partial_{K} e_{b}{ }^{M} \partial_{L} e^{b N}+\left(\mathcal{H}^{N K}-\eta^{N K}\right)\left(\mathcal{H}_{M L}+\eta_{M L}\right) \partial_{K} e_{b}{ }^{M} \partial^{L} e^{b}{ }_{N} .
\end{aligned}
$$


We next compute with (2.32)

$$
\Omega_{[a b c]}^{2}=9 f_{[a b c]}^{2}=3\left(f_{a b c}+2 f_{c a b}\right) f^{a b c},
$$

where (5.7) has been used. This yields

$$
\begin{aligned}
\Omega_{[a b c]}^{2} & =3\left[\frac{1}{4} \mathcal{H}^{K L}\left(\mathcal{H}_{M N}-\eta_{M N}\right) \partial_{K} e_{b}{ }^{M} \partial_{L} e^{b N}+2\left(e_{c} e_{a}{ }^{M}\right) e_{b M}\left(e^{a} e^{b N}\right) e^{c}{ }_{N}\right] \\
& =\frac{3}{4}\left[\mathcal{H}_{K L}\left(\mathcal{H}_{M N}-\eta_{M N}\right)-2\left(\mathcal{H}_{M L}-\eta_{M L}\right)\left(\mathcal{H}_{N K}-\eta_{N K}\right)\right] \partial^{K} e_{b}{ }^{M} \partial^{L} e^{b N},
\end{aligned}
$$

where we used in the second line (5.7). In total, the third and the fourth term of $\mathcal{R}$ in (5.8) combine as follows:

$$
\begin{aligned}
-\frac{1}{4} \Omega_{a b \bar{c}}{ }^{2}-\frac{1}{12} \Omega_{[a b c]}{ }^{2}= & \frac{1}{8} \mathcal{H}^{K L}\left(\mathcal{H}_{M N}+\eta_{M N}\right) \partial_{K} e_{b}{ }^{M} \partial_{L} e^{b N}+\frac{1}{4}\left(\mathcal{H}_{M N}+\eta_{M N}\right) e_{b} e_{a}{ }^{M} e^{a} e^{b N} \\
& -\frac{1}{8}\left(\mathcal{H}^{N K}-\eta^{N K}\right)\left(\mathcal{H}_{M L}+3 \eta_{M L}\right) \partial_{K} e_{b}{ }^{M} \partial^{L} e^{b}{ }_{N}
\end{aligned}
$$

Adding (5.11) and (5.17) one obtains after some work

$$
\left.\mathcal{R}\right|_{\mathcal{H}}=-\frac{1}{4} \partial_{M} \partial_{N} \mathcal{H}^{M N}+\frac{1}{32} \mathcal{H}^{K L} \partial_{K} \mathcal{H}_{M N} \partial_{L} \mathcal{H}^{M N}-\frac{1}{8} \mathcal{H}^{M L} \partial_{K} \mathcal{H}_{M N} \partial_{L} \mathcal{H}^{N K}
$$

In combination with (5.10) we obtain in total

$$
\begin{aligned}
\mathcal{R}= & \mathcal{H}^{M N} \partial_{M} \partial_{N} d+\partial_{N} \mathcal{H}^{M N} \partial_{M} d-\mathcal{H}^{M N} \partial_{M} d \partial_{N} d \\
& -\frac{1}{4} \partial_{M} \partial_{N} \mathcal{H}^{M N}+\frac{1}{32} \mathcal{H}^{K L} \partial_{K} \mathcal{H}^{M N} \partial_{L} \mathcal{H}_{M N}-\frac{1}{8} \mathcal{H}^{M L} \partial_{L} \mathcal{H}^{N K} \partial_{K} \mathcal{H}_{M N} .
\end{aligned}
$$

This coincides with the curvature scalar $\mathcal{R}(\mathcal{H}, d)$ constructed in [9], up to the same irrelevant overall factor of 4 encountered above, and thus we have established independently the equivalence of the two action principles.

\section{$5.3 \quad \mathcal{H}$-compatible Christoffel connections}

So far we have exclusively dealt with covariant derivatives acting on objects with 'flat' or tangent space indices. Given these spin connection-type objects there is a canonical way to associate corresponding Christoffel-type connections, via the so-called vielbein postulate. Here we investigate the properties of these Christoffel symbols. Very recently, an interesting paper appeared that deals with the geometrical foundation of the $\mathcal{H}$-formulation and introduces similar connections [14], which are related but not identical to those discussed here.

We start by defining Christoffel symbols from the $G L(D) \times G L(D)$ connections by requiring

$$
\nabla_{M} V_{N}:=\partial_{M} V_{N}-\Gamma_{M N}{ }^{K} V_{K} \equiv e_{M}^{A} e_{N}^{B} \nabla_{A} V_{B}
$$

and analogously for higher tensors. This is satisfied if the following 'vielbein postulate' holds

$$
\partial_{M} e_{N}^{A}-\omega_{M B}{ }^{A} e_{N}^{B}-\Gamma_{M N}{ }^{K} e_{K}^{A}=0
$$


which is the usual condition that the vielbein is covariantly constant with respect to the tangent space and Christoffel connections. This condition determines the Christoffel symbols in terms of (derivatives of) $e$ and $\omega$. Thus, $\Gamma$ is uniquely determined by the physical fields whenever this holds for $\omega$. Moreover, as the conventional torsion for $\omega$ is non-zero, there is a non-zero antisymmetric part $\Gamma_{[M N]}^{K}$ proportional to this torsion. With this covariant derivative curvature tensors may be defined via $\left[\nabla_{M}, \nabla_{N}\right]$, and the resulting objects will be equivalent (through the conversion of indices with the frame field) to the corresponding tensors defined via (2.41), and thus all the comments there readily apply in the present context.

It is instructive, however, to inspect some properties of the covariant derivatives based on $\Gamma$ in more detail. First, from (5.21) we infer the transformation rule of $\Gamma$ under $\xi^{M}$ transformations,

$$
\delta_{\xi} \Gamma_{M N}{ }^{K}=\widehat{\mathcal{L}}_{\xi} \Gamma_{M N}{ }^{K}+\partial_{M} \partial_{N} \xi^{K}-\partial_{M} \partial^{K} \xi_{N} .
$$

One may easily verify that this is the right transformation rule that makes the first expression in (5.20) a covariant derivative. The first inhomogeneous term is the standard one appearing for the Christoffel symbols in Riemannian geometry, while the second one is novel and due to the generalized Lie derivative. This new contribution also shows that $\delta_{\xi} \Gamma_{[M N]}{ }^{K} \neq 0$ and thus that the connection is necessarily torsionful.

An important consequence of the defining relation (5.20) is that $\eta_{M N}$ is covariantly constant,

$$
\nabla_{M} \eta_{N K}=e_{M}{ }^{A} e_{N}{ }^{B} e_{K}{ }^{C} \nabla_{A} \mathcal{G}_{B C}=0,
$$

which follows from the definition (2.11) and the metricity condition (2.27) for the tangent space metric. Explicitly, this implies for the Christoffel symbols with (5.20)

$$
\nabla_{M} \eta_{N K}=\partial_{M} \eta_{N K}-\Gamma_{M N}{ }^{L} \eta_{L K}-\Gamma_{M K}{ }^{L} \eta_{N L}=0 \quad \Rightarrow \quad \Gamma_{M(N K)}=0 .
$$

Moreover, since the 'flattened' components of $\mathcal{H}$ are given by the components of $\mathcal{G}^{A B}$, up to sign differences that account for the different signatures, c.f. eq. (5.3), the metricity of the tangent space metric implies also

$$
\nabla_{M} \mathcal{H}_{N K}=e_{M}{ }^{A} e_{N}{ }^{B} e_{K}^{C} \nabla_{A} \mathcal{H}_{B C}=0
$$

and therefore $\Gamma$ is an $\mathcal{H}$-compatible connection. In other words, in this formalism there are two covariantly constant metrics, $\mathcal{H}$ and $\eta$.

Another important property of the Christoffel symbols follows from the vielbein postulate (5.21),

$$
\Gamma_{[M N K]}=-f_{[M N K]}-\omega_{[M N K]}=0,
$$

where all indices have been converted into world indices, and the last equation follows from (2.32). As the latter equation was a direct consequence of the (generalized) torsion constraint, eq. (5.26) can be seen as the analogue of the usual torsion constraint $\Gamma_{[M N] K}=0$. The properties (5.24) and (5.26) imply that in the generalized Lie derivatives the partial derivatives can be replaced by covariant derivatives [14,

$$
\begin{aligned}
\widehat{\mathcal{L}}_{\xi} V_{M} & =\xi^{N} \partial_{N} V_{M}+\left(\partial_{M} \xi^{N}-\partial^{N} \xi_{M}\right) V_{N} \\
& =\xi^{N} \nabla_{N} V_{M}+\left(\nabla_{M} \xi^{N}-\nabla^{N} \xi_{M}\right) V_{N}+\xi^{N} V^{K}\left(\Gamma_{N M K}-\Gamma_{M N K}-\Gamma_{K M N}\right) \\
& =\xi^{N} \nabla_{N} V_{M}+\left(\nabla_{M} \xi^{N}-\nabla^{N} \xi_{M}\right) V_{N},
\end{aligned}
$$


and similarly on arbitrary higher tensors. In conventional Riemannian geometry the usual Lie derivative has the analogous property by virtue of the usual torsion constraint.

The frame formalism carries only connections with respect to $G L(D) \times G L(D)$, and so this 'factorization' should also be visible in the Christoffel symbols of the generalized metric formulation. To see this, we note that due to the constraint (5.2) on $\mathcal{H}$, the matrices

$$
\mathcal{P}_{M}{ }^{N}=\frac{1}{2}\left(\delta_{M}^{N}-\mathcal{H}_{M}{ }^{N}\right), \quad \overline{\mathcal{P}}_{M}{ }^{N}=\frac{1}{2}\left(\delta_{M}^{N}+\mathcal{H}_{M}{ }^{N}\right)
$$

are projectors satisfying $\mathcal{P}^{2}=\mathcal{P}$ and $\overline{\mathcal{P}}^{2}=\overline{\mathcal{P}}$. With the expression (5.1) for $\mathcal{H}$ in terms of the frame fields, this simply reduces to [9]

$$
\mathcal{P}_{M}{ }^{N}=e_{a M} e^{a N}, \quad \overline{\mathcal{P}}_{M}{ }^{N}=e_{\bar{a} M} e^{\bar{a} N} .
$$

Therefore, $\overline{\mathcal{P}}$ and $\mathcal{P}$ project onto the subspaces that are invariant under the left $G L(D)$ or the right $G L(D)$, respectively. More precisely, given a vector $V_{M}$ that is projected to the left ('unbarred') subspace, i.e., invariant under the right $G L(D)$, we find indeed

$$
V_{M}=\mathcal{P}_{M}^{N} V_{N}=e_{a M} e^{a N} V_{N} \quad \Rightarrow \quad V_{\bar{a}} \equiv e_{\bar{a}}^{M} V_{M}=0
$$

and thus only $V_{a}$ is non-zero. Here, in the last step, (2.13) has been used. Analogously, the frame components of a vector with $V_{M}=\overline{\mathcal{P}}_{M}{ }^{N} V_{N}$ satisfy $V_{a}=0$.

It is now straightforward to see that the covariant derivative is compatible with these projections. In fact, since $\mathcal{H}$ and $\eta$ are covariantly constant, we find

$$
V_{M}=\mathcal{P}_{M}{ }^{N} V_{N} \quad \Rightarrow \quad \nabla_{M} V_{N}=\mathcal{P}_{N}{ }^{K} \nabla_{M} V_{K},
$$

and similarly for $\overline{\mathcal{P}}$. This is the analogue of the fact, which is manifest in the frame formalism, that the $G L(D) \times G L(D)$ covariant derivatives preserve the barred-unbarred index structure.

The Christoffel symbols discussed here are closely related to those introduced in [14]. First, the property that the covariant derivatives preserve the left- and right-invariant subspaces as in (5.31) is one of the requirements that determines their $\Gamma$. Second, the Christoffel symbols are further constrained in [14] by requiring (5.24) and (5.26). The details of the connections differ, however, in that their covariant derivatives do not transform covariantly, but only in certain combinations and projections, while Siegel's connections - and thereby the Christoffel symbols determined by (5.21) - properly transforms as connections, at the cost of introducing components that are not determined in terms of the physical fields.

\section{Summary and Outlook}

Recent results on double field theory have given an elegant ' $O(D, D)$ covariantisation' of the conventional low-energy space-time action (1.1) of closed string theory by virtue of introducing extra coordinates. The resulting actions, written in terms of $\mathcal{E}_{i j}$ or $\mathcal{H}^{M N}$, take a remarkably simple form and feature besides the global $O(D, D)$ T-duality invariance a gauge symmetry that unifies the usual 
diffeomorphisms with the 2-form gauge symmetry. So far, however, a deeper understanding of the geometrical structure of this theory, adopting the role that Riemannian geometry plays in Einstein's theory, was lacking. In this paper we have shown that the duality-covariant geometrical formalism developed by Siegel already some time ago in [16] provides, at least to some extent, such a framework in terms of frame fields, connections and curvatures for the gauge group $G L(D) \times G L(D)$. For the convenience of the reader we summarize here the main differences to ordinary Riemannian geometry.

First of all, a central object is the $O(D, D)$ invariant metric $\eta$ which is a constant 'world tensor' with two upper or two lower indices. In Riemannian geometry such an object would not be welldefined, but here the constancy of $\eta$ has a gauge invariant meaning due to the modified form of the gauge transformations, governed by the 'generalized Lie derivatives' (2.1). In contrast to the 'world' metric $\eta^{M N}$, the 'tangent space metric' $\mathcal{G}_{A B}$ is space-time dependent, and thus we have the opposite of the usual situation. It is instructive to compare this with a reformulation of conventional Riemannian geometry that resembles the formalism presented here in that there is an enlarged group of frame transformations, the general linear group $G L(D)$ rather than the Lorentz group, and a space-time dependent tangent space metric $g_{a b}$ that enters together with the vielbein $e_{a}{ }^{m}$ as an independent field (see sec. IX.A.2 in [22]). Imposing a metricity condition and the usual torsion constraint,

$$
\nabla_{a} g_{b c}=0, \quad T_{a b}{ }^{c}=-2 e_{a}{ }^{m} e_{b}{ }^{n} \nabla_{[m} e_{n]}^{c}=0
$$

allows one to solve for the connections $\omega_{a b c}$ in terms of derivatives of $e_{a}{ }^{m}$ and $g_{a b}$. The local $G L(D)$ symmetry can then be fixed by setting either $e_{a}{ }^{m}=\delta_{a}{ }^{m}$, in which case $g_{a b}$ can be identified with the usual metric and the $\omega_{a b c}$ reduce to the Christoffel symbols $\Gamma_{a b c}$, or one can set $g_{a b}=\delta_{a b}$, in which case $e_{a}{ }^{m}$ carries the physical degrees of freedom and $\omega_{a b c}$ reduces to the usual spin connection. This formalism differs, however, from the present frame formalism, at least in the form discussed in this paper, in several respects. For instance, here it is not the tangent space metric $\mathcal{G}_{A B}$ that is introduced as an independent object but rather the constant $O(D, D)$ invariant metric $\eta^{M N}$, while $\mathcal{G}_{A B}$ is defined in terms of $\eta_{M N}$ by use of the frame fields. Moreover, the torsion constraint is modified as compared to (6.1).

Perhaps the most important difference to Riemannian geometry is the novel gauge symmetry parametrized by $\xi^{M}$, whose algebra is governed by the C-bracket rather than the Lie bracket of the usual diffeomorphisms. This has a number of consequences. Most importantly, due to the modified torsion constraint, the Riemann-like tensor defined through the commutator of covariant derivatives is generally not covariant under frame rotations. Following [16] this can be repaired 'by hand', but is should be stressed that the resulting tensor, which is fully covariant, is not in all components independent on the undetermined connections. The resulting Ricci-like tensor and scalar curvature are, however, fully expressible in terms of the physical fields, and are equivalent to the field equations and Lagrangian of double field theory, respectively.

It is natural to anticipate that a yet better understanding of the geometrical structure is possible, perhaps adopting and extending ideas from 'generalized geometry' [18, 19, 20], in which, for instance, a fully covariant curvature tensor may emerge more directly. Such an understanding could be useful not only for the double field theory currently discussed, but also for further generalizations, say, to type II 
string theory. Finally, we hope that the present investigations might shed some light on the possibility of the ultimate goal of this research program, namely to construct a 'truly doubled field theory' in which the strong constraint (1.7) is relaxed in such a way that the fields may depend non-trivially on both momentum and winding coordinates even locally.

\section{Acknowledgments}

We are happy to acknowledge helpful discussions with Ashoke Sen and especially Barton Zwiebach. We have also benefitted from discussions with Chris Hull at initial stages of this project and later correspondence. This work is supported by the U.S. Department of Energy (DoE) under the cooperative research agreement DE-FG02-05ER41360. The work of OH is supported by the DFG - The German Science Foundation. The work of SK is supported in part by a Samsung Scholarship.

\section{A Computational details on the curvature tensor}

\section{A.1 Fully contracted curvature tensor without constraints}

In this appendix we prove the equation (2.56) which holds before imposing any constraints. Thus, we have to use the form of the curvature tensor in (2.50), which was fully covariant without using constraints. More explicitly, this reads

$$
\begin{aligned}
\mathcal{R}_{A B C D}= & \frac{1}{2}\left[R_{A B C D}+R_{C D A B}\right] \\
& -\frac{1}{4}\left[\omega_{E C D} \omega^{E}{ }_{A B}+\omega_{E A B} \omega^{E}{ }_{C D}\right]-\frac{1}{4}\left[\omega_{E C D} e^{E} \mathcal{G}_{A B}+\omega_{E A B} e^{E} \mathcal{G}_{C D}\right],
\end{aligned}
$$

from which we derive

$$
\mathcal{R}_{A B}{ }^{A B}=\mathcal{G}^{A C} \mathcal{G}^{B D} \mathcal{R}_{A B C D}=\mathcal{G}^{A C} R_{A B C}{ }^{B}-\frac{1}{2} \omega_{E}{ }^{A B} e^{E} \mathcal{G}_{A B}-\frac{1}{2} \omega_{E}{ }^{A B} \omega^{E}{ }_{A B}
$$

We rewrite now the first term on the right-hand side as follows

$$
\begin{aligned}
R_{A B}{ }^{A B} & =\mathcal{G}^{A C}\left(e_{A} \omega_{B C}{ }^{B}-e_{B} \omega_{A C}{ }^{B}+\omega_{A C}{ }^{E} \omega_{B E}{ }^{B}-\omega_{B C}{ }^{E} \omega_{A E}{ }^{B}-\Omega_{A B}{ }^{E} \omega_{E C}{ }^{B}\right) \\
& =\mathcal{G}^{A C}\left(\nabla_{A} \omega_{B C}{ }^{B}-\nabla_{B} \omega_{A C}{ }^{B}-\omega_{B E}{ }^{B} \omega_{A C}{ }^{E}\right)+\left(-\Omega_{A B}{ }^{E}+\omega_{B A}{ }^{E}\right) \omega_{E}{ }^{A B} .
\end{aligned}
$$

In the first bracket we next move the metrics inside the covariant derivatives, where we have to remember that here the metric is not assumed to be covariantly constant,

$$
\begin{aligned}
\mathcal{G}^{A C}\left(\nabla_{A} \omega_{B C}{ }^{B}-\nabla_{B} \omega_{A C}{ }^{B}-\omega_{B E}{ }^{B} \omega_{A C}{ }^{E}\right)= & \nabla_{A} \omega_{B}{ }^{A B}-\omega_{B C}{ }^{B} \nabla_{A} \mathcal{G}^{A C}-\nabla_{B} \omega_{A}{ }^{A B} \\
& +\omega_{A C}{ }^{B} \nabla_{B} \mathcal{G}^{A C}-\omega_{B E}{ }^{B} \omega_{A}{ }^{A E} .
\end{aligned}
$$

In order to eliminate the last term proportional to $\omega^{2}$, we use

$$
\begin{aligned}
\nabla_{A} \nabla_{B} \mathcal{G}^{A B}= & e_{A} e_{B} \mathcal{G}^{A B}-\nabla_{A} \omega_{B}^{B A}-\nabla_{A} \omega_{B}{ }^{A B} \\
& -\omega_{A C}{ }^{A} \nabla_{B} \mathcal{G}^{C B}-\omega_{B E}{ }^{B} \omega_{A}{ }^{A E}-\omega_{B E}{ }^{B} \omega_{A}{ }^{E A}
\end{aligned}
$$


which is straightforward to verify. This leads to

$$
\begin{aligned}
\mathcal{G}^{A C}\left(\nabla_{A} \omega_{B C}{ }^{B}-\nabla_{B} \omega_{A C}{ }^{B}-\omega_{B E}{ }^{B} \omega_{A C}{ }^{E}\right)= & 2 \nabla_{A} \omega_{B}{ }^{A B}+\nabla_{A} \nabla_{B} \mathcal{G}^{A B}-e_{A} e_{B} \mathcal{G}^{A B} \\
& +\omega_{A C}{ }^{B} \nabla_{B} \mathcal{G}^{A C}+\omega_{B E}{ }^{B} \omega_{A}{ }^{E A} .
\end{aligned}
$$

We use next the definition $\tilde{T}_{A}=\omega_{B A}{ }^{B}+f_{B A}{ }^{B}-2 e_{A} d$ in order to eliminate $\omega_{B A}{ }^{B}$ in the first and last term by $\tilde{T}$ and $f$. Moreover, we use $2 f_{A(B C)}=e_{A} \mathcal{G}_{B C}$ in order to rewrite the third term in terms of $f$. This leads after a short computation to

$$
\begin{aligned}
\mathcal{G}^{A C} & \left(\nabla_{A} \omega_{B C}{ }^{B}-\nabla_{B} \omega_{A C}{ }^{B}-\omega_{B E}{ }^{B} \omega_{A C}{ }^{E}\right) \\
= & 2 \nabla_{A} \tilde{T}^{A}+2 e_{A} f_{B}{ }^{[A B]}+2 \omega_{A E}{ }^{A} f_{B}{ }^{E B}+\nabla_{A} \nabla_{B} \mathcal{G}^{A B}+\omega_{A C}{ }^{B} \nabla_{B} \mathcal{G}^{A C} \\
& +\tilde{T}_{A}{ }^{2}+f_{B E}{ }^{B} f_{A}{ }^{E A}-2 \tilde{T}_{A} f_{B}{ }^{A B}-4 f_{B E}{ }^{B} e^{E} d+4\left(\nabla_{A} e^{A} d+\tilde{T}_{A} e^{A} d\right),
\end{aligned}
$$

where we have used the constraint (2.15). In here, the terms in brackets in the last line vanish by (2.39). By finally eliminating $\omega_{A E}{ }^{A}$ in the third term above in terms of $\tilde{T}$ and $f$, we arrive at

$$
\begin{aligned}
\mathcal{G}^{A C} & \left(\nabla_{A} \omega_{B C}{ }^{B}-\nabla_{B} \omega_{A C}{ }^{B}-\omega_{B E}{ }^{B} \omega_{A C}{ }^{E}\right) \\
& =2 \nabla_{A} \tilde{T}^{A}+\tilde{T}_{A}^{2}+\nabla_{A} \nabla_{B} \mathcal{G}^{A B}+2 e_{B} f_{A}^{[A B]}-f_{B E}{ }^{B} f_{A}{ }^{E A}+\omega_{A C}{ }^{B} \nabla_{B} \mathcal{G}^{A C} .
\end{aligned}
$$

Using this in (A.3) we get

$$
\begin{aligned}
R_{A B}{ }^{A B}= & 2 \nabla_{A} \tilde{T}^{A}+\tilde{T}_{A}^{2}+\nabla_{A} \nabla_{B} \mathcal{G}^{A B} \\
& +2 e_{B} f_{A}^{[A B]}-f_{B E}{ }^{B} f_{A}{ }^{E A}+\omega_{A C}{ }^{B} \nabla_{B} \mathcal{G}^{A C}+\left(-\Omega_{A B}{ }^{E}+\omega_{B A}{ }^{E}\right) \omega_{E}{ }^{A B} .
\end{aligned}
$$

To proceed with the computation of the full $\mathcal{R}_{A B}{ }^{A B}$ in (A.2) we define

$$
\begin{aligned}
\Delta \mathcal{R} \equiv & 2 e_{B} f_{A}{ }^{[A B]}-f_{B E}{ }^{B} f_{A}{ }^{E A}+\omega_{A C}{ }^{B} \nabla_{B} \mathcal{G}^{A C}+\left(-\Omega_{A B}{ }^{E}+\omega_{B A}{ }^{E}\right) \omega_{E}{ }^{A B} \\
& -\frac{1}{2} \omega_{E}{ }^{A B} e^{E} \mathcal{G}_{A B}-\frac{1}{2} \omega_{E}{ }^{A B} \omega^{E}{ }_{A B},
\end{aligned}
$$

such that

$$
\mathcal{R}_{A B}{ }^{A B}=2 \nabla_{A} \tilde{T}^{A}+\tilde{T}_{A}^{2}+\nabla_{A} \nabla_{B} \mathcal{G}^{A B}+\Delta \mathcal{R}
$$

To evaluate $\Delta \mathcal{R}$, we need the following identities:

$$
\begin{aligned}
2 e_{B} f_{A}[A B] & =f_{B E}{ }^{B} f_{A}{ }^{E A}-f_{A B}{ }^{E} f_{E}{ }^{A B} \\
\nabla_{A} \mathcal{G}_{B C} & =e_{A} \mathcal{G}_{B C}+\omega_{A B C}+\omega_{A C B}=2 f_{A(B C)}+2 \omega_{A(B C)} .
\end{aligned}
$$

Using the definition $\Omega_{A B}{ }^{C}=2 f_{[A B]}^{C}+f_{[A B]}^{C}$, we can rewrite $\Delta \mathcal{R}$ in terms of only $f$ and $\omega$ as follows:

$$
\Delta \mathcal{R}=-\left(f_{A B E} f^{E A B}+2 \omega_{A B E} f^{E A B}+\omega_{A B E} \omega^{E A B}\right)-\frac{1}{2}\left(2 \omega^{E A B} f_{E A B}+\omega^{E A B} \omega_{E A B}\right) .
$$

By adding $-\frac{1}{2} f^{E A B} f_{E A B}$, which vanishes due to the strong constraint, $\Delta \mathcal{R}$ can be written as

$$
\Delta \mathcal{R}=-K^{E A B} K_{A B E}-\frac{1}{2} K^{E A B} K_{E A B}=-\frac{1}{2} K^{E A B}\left(K_{A B E}+K_{B E A}+K_{E A B}\right),
$$


where we introduced

$$
K_{A B}{ }^{C}=f_{A B}^{C}+\omega_{A B}{ }^{C} .
$$

We can further rewrite $\Delta \mathcal{R}$ according to

$$
\Delta \mathcal{R}=-\frac{3}{2} K_{[A B C]}^{2}-\frac{3}{2} K_{(A B C)}{ }^{2} .
$$

Next, we use the following two identities

$$
\mathcal{T}_{[A B C]}=3 K_{[A B C]}, \quad K_{(A B C)}=\frac{1}{2} \nabla_{(A} \mathcal{G}_{B C)},
$$

which can be easily confirmed, to obtain

$$
\Delta \mathcal{R}=-\frac{1}{6} \mathcal{T}_{[A B C]}{ }^{2}-\frac{3}{8} \nabla_{(A} \mathcal{G}_{B C)}{ }^{2} .
$$

This finally leads to

$$
\mathcal{R}_{A B}{ }^{A B}=2 \nabla_{A} \tilde{T}^{A}+\tilde{T}_{A}{ }^{2}+\nabla_{A} \nabla_{B} \mathcal{G}^{A B}-\frac{1}{6} \mathcal{T}_{[A B C]}{ }^{2}-\frac{3}{8} \nabla_{\left({ }_{A} \mathcal{G}_{B C)}{ }^{2}\right.},
$$

as we wanted to show.

\section{A.2 Scalar curvature}

In this appendix we prove that the curvature scalar given by the first expression in (2.58) can be written as (2.60). From the definition of the Riemann tensor (2.48) we obtain

$$
\mathcal{R}=-\frac{1}{2} \mathcal{R}_{a b}{ }^{a b}=-\frac{1}{2} R_{a b}{ }^{a b}-\frac{1}{4} \omega_{E a b} \omega^{E b a},
$$

where $R_{a b}^{a b}$ is given by

$$
\begin{aligned}
R_{a b}{ }^{a b} & =\mathcal{G}^{a c}\left[e_{a} \omega_{b c}{ }^{b}-e_{b} \omega_{a c}{ }^{b}+\omega_{a c}{ }^{e} \omega_{b e}{ }^{b}-\omega_{b c}{ }^{e} \omega_{a e}{ }^{b}-\Omega_{a b}{ }^{E} \omega_{E c}{ }^{b}\right] \\
& =-e_{a} \tilde{\Omega}^{a}+\left(e_{a} \mathcal{G}^{a c}\right) \tilde{\Omega}_{c}-e_{b}\left(\mathcal{G}^{a c} \omega_{a c}^{b}\right)+\left(e_{b} \mathcal{G}^{a c}\right) \omega_{a c}^{b}+\omega_{a}^{a e} \omega_{b e}^{b}-\omega_{b}{ }^{a e} \omega_{a e}{ }^{b}-\Omega_{a b}{ }^{E} \omega_{E}{ }^{a b}
\end{aligned}
$$

Using

$$
\omega_{a}^{a b}=\tilde{\Omega}^{b}-\mathcal{G}^{b c} e^{a} \mathcal{G}_{a c}=\tilde{\Omega}^{b}+e_{a} \mathcal{G}^{a b},
$$

which follows from (2.37) and (2.29), a short computation shows that this can be rewritten as

$$
R_{a b}{ }^{a b}=-2 e_{a} \tilde{\Omega}^{a}-\tilde{\Omega}_{a}{ }^{2}-e_{a} e_{b} \mathcal{G}^{a b}+\left[\omega_{b c a} \omega^{a c b}-\Omega_{a b}{ }^{E} \omega_{E}^{a b}\right] .
$$

Then $\mathcal{R}$ is according to (A.21) given by

$$
\mathcal{R}=-\frac{1}{2} \mathcal{R}_{a b}{ }^{a b}=e_{a} \tilde{\Omega}^{a}+\frac{1}{2} \tilde{\Omega}_{a}{ }^{2}+\frac{1}{2} e_{a} e_{b} \mathcal{G}^{a b}-\frac{1}{2}\left[\omega_{b c a} \omega^{a c b}-\Omega_{a b}{ }^{E} \omega_{E}{ }^{a b}+\frac{1}{2} \omega_{E a b} \omega^{E b a}\right] .
$$

We next evaluate the terms in the square bracket. Using the torsion constraint (2.30) we find

$$
\begin{aligned}
\omega_{b c a} \omega^{a c b}-\Omega_{a b}{ }^{E} \omega_{E}^{a b}+\frac{1}{2} \omega_{E a b} \omega^{E b a} & =\omega_{a b c} \omega^{c a b}+\frac{1}{2} \omega_{c a b} \omega^{c a b}+\frac{1}{2} \omega_{\bar{c} a b} \omega^{\bar{c} a b} \\
& =\frac{1}{2}\left[\omega_{a b c}+\omega_{b c a}+\omega_{c a b}\right] \omega^{c a b}+\frac{1}{2} \omega_{\bar{c} a b} \omega^{\bar{c} a b}
\end{aligned}
$$


where in the second equation $\omega_{b c a} \omega^{c a b}=\omega_{a b c} \omega^{c a b}$ has been used. The terms in the square bracket in the second line can be written as

$$
\begin{aligned}
\frac{1}{2}\left[\omega_{a b c}+\omega_{b c a}+\omega_{c a b}\right] \omega^{c a b}= & \frac{1}{4}\left[\omega_{a b c}+\omega_{b c a}+\omega_{c a b}-\omega_{a c b}-\omega_{b a c}-\omega_{c b a}\right] \omega^{c a b} \\
& +\frac{1}{4}\left(\omega_{c a b}+\omega_{c b a}\right) \omega^{c a b}+\frac{1}{4}\left(\omega_{a b c}+\omega_{b c a}+\omega_{a c b}+\omega_{b a c}\right) \omega^{c a b} .
\end{aligned}
$$

The terms in the each bracket give the following contributions, respectively:

$$
\begin{aligned}
\frac{3}{2} \omega_{[a b c]} \omega^{[a b c]} & =\frac{1}{6} \Omega_{[a b c]}{ }^{2}=\frac{3}{2} f_{[a b c]} f^{[a b c]}, \\
\frac{1}{2} \omega_{c(a b)} \omega^{c(a b)} & =-\frac{1}{8} e^{a} \mathcal{G}^{b c} e_{a} \mathcal{G}_{b c}=\frac{1}{2} f_{c(a b)} f^{c(a b)}, \\
\frac{1}{4}\left(\omega_{a b c}+\omega_{b c a}+\omega_{a c b}+\omega_{b a c}\right) \omega^{c a b} & =\omega_{a(b c)} \omega^{b(a c)}=-\frac{1}{4} e^{a} \mathcal{G}^{b c} e_{b} \mathcal{G}_{a c}=f_{a(b c)} f^{b(a c)} .
\end{aligned}
$$

Thus, (A.26) can finally be written as

$$
\omega_{b c a} \omega^{a c b}-\Omega_{a b}{ }^{E} \omega_{E}^{a b}+\frac{1}{2} \omega_{E a b} \omega^{E b a}=\frac{1}{6} \Omega_{[a b c]}{ }^{2}-\frac{1}{8} e^{a} \mathcal{G}^{b c} e_{a} \mathcal{G}_{b c}-\frac{1}{4} e^{a} \mathcal{G}^{b c} e_{b} \mathcal{G}_{a c}+\frac{1}{2} \omega_{\bar{c} a b} \omega^{\bar{c} a b} .
$$

Using this in (A.25) together with $\omega_{\bar{c} a b}=-\Omega_{\bar{c} a b}$ we arrive at

$$
\mathcal{R}=e_{a} \tilde{\Omega}^{a}+\frac{1}{2} \tilde{\Omega}_{a}^{2}+\frac{1}{2} e_{a} e_{b} \mathcal{G}^{a b}-\frac{1}{12} \Omega_{[a b c]}{ }^{2}+\frac{1}{16} e^{a} \mathcal{G}^{b c} e_{a} \mathcal{G}_{b c}+\frac{1}{8} e^{a} \mathcal{G}^{b c} e_{b} \mathcal{G}_{a c}-\frac{1}{4} \Omega_{\bar{c} a b} \Omega^{\bar{c} a b}
$$

With the expressions in terms of $f$ in (A.28), (A.29) and (A.30) this can also be written as

$$
\mathcal{R}=e_{a} \tilde{\Omega}^{a}+\frac{1}{2} \tilde{\Omega}_{a}^{2}+\frac{1}{2} e_{a} e_{b} \mathcal{G}^{a b}-\frac{1}{4}\left(f_{a b c}+f_{b c a}+f_{c a b}\right) f^{c a b}-\frac{1}{4} \Omega_{\bar{c} a b} \Omega^{\bar{c} a b} .
$$

The expressions for $\mathcal{R}$ given so far can be further simplified by using for the last term in (A.32) the following relation

$$
\Omega_{\bar{c} a b}=\Omega_{a b \bar{c}}+\frac{1}{2} e_{\bar{c}} \mathcal{G}_{a b}
$$

which implies

$$
\frac{1}{2} \Omega_{\bar{c} a b}^{2}=\frac{1}{2} \Omega_{a b \bar{c}}^{2}-\frac{1}{8} e^{\bar{c}} \mathcal{G}^{a b} e_{\bar{c}} \mathcal{G}_{a b}=\frac{1}{2} \Omega_{a b \bar{c}}{ }^{2}+\frac{1}{8} e^{c} \mathcal{G}^{a b} e_{c} \mathcal{G}_{a b} .
$$

Here we used that the mixed term in the square can be brought to the form of the last term, using $\Omega_{\bar{c}(a b)}=\frac{1}{2} e_{\bar{c}} \mathcal{G}_{a b}$. In total, using (A.35) in (A.32) yields the following expression for the scalar curvature:

$$
\mathcal{R}=e_{a} \tilde{\Omega}^{a}+\frac{1}{2} \tilde{\Omega}_{a}^{2}+\frac{1}{2} e_{a} e_{b} \mathcal{G}^{a b}-\frac{1}{4} \Omega_{a b \bar{c}}^{2}-\frac{1}{12} \Omega_{[a b c]}{ }^{2}+\frac{1}{8} e^{a} \mathcal{G}^{b c} e_{b} \mathcal{G}_{a c},
$$

as we wanted to show.

\section{A.3 Scalar curvature in terms of $G L(D) \times G L(D)$ covariant derivatives}

Here we derive from the expression (A.32) for the scalar curvature the manifestly $O(D, D)$ and $G L(D) \times$ $G L(D)$ covariant form given in (2.59). We first focus on the dilaton-dependent terms which originate 
only from the first two terms in (A.32),

$$
\begin{aligned}
\left.\mathcal{R}\right|_{d} & =e_{a}\left(-2 e^{a} d\right)+\frac{1}{2}\left[2 \mathcal{G}^{a b}\left(\partial_{M} e_{a}^{M}\right)\left(-2 e_{b} d\right)+\left(-2 e_{a} d\right)\left(-2 e^{a} d\right)\right] \\
& =-2\left(e_{a} e^{a} d+e^{2 d} \partial_{M}\left(e_{a}{ }^{M} e^{-2 d}\right) e^{a} d+e_{a} d e^{a} d\right) \\
& =-2\left(e_{a} e^{a} d-\omega_{b a}{ }^{b} e^{a} d+e_{a} d e^{a} d\right)=-2 \nabla_{a} \nabla^{a} d-2 \nabla_{a} d \nabla^{a} d .
\end{aligned}
$$

This reproduces the terms in (2.59) that are quadratic in the dilaton, if we use the strong constraint in the forms (2.15) and (2.39). There are also apparent terms linear in the dilaton in (2.59) but these are actually artifacts of the dilaton dependence of some of the covariant derivatives. More precisely, terms in the first and last line of (2.59) can be combined as follows

$$
\begin{aligned}
& -\frac{1}{2}\left(\nabla^{a}\left(e_{a}{ }^{M} \bar{\nabla}^{\bar{b}} e_{\bar{b} M}\right)-\bar{\nabla}^{\bar{a}}\left(e_{\bar{a}}^{M} \nabla^{b} e_{b M}\right)\right)-\left(\nabla^{a} d\left(e_{a}{ }^{M} \bar{\nabla}^{\bar{b}} e_{\bar{b} M}\right)-\bar{\nabla}^{\bar{a}} d\left(e_{\bar{a}}^{M} \nabla^{b} e_{b M}\right)\right) \\
& =-\frac{1}{2} \partial_{M}\left(e_{a}{ }^{M} e^{a N} e^{\bar{b}} e_{\bar{b} N}-e_{\bar{a}}{ }^{M} e^{\bar{a} N} e^{b} e_{b N}\right) .
\end{aligned}
$$

This shows that this expression is independent of the dilaton and thus we have shown that (A.32) reproduces the correct dilaton-dependent terms. For the following computation it is convenient to simplify the structure obtained in (A.38) further, which yields

$$
\begin{aligned}
\frac{1}{2} \partial_{M}\left(e_{\bar{a}}^{M} e^{\bar{a} N} e^{b} e_{b N}-e_{a}^{M} e^{a N} e^{\bar{b}} e_{\bar{b} N}\right) & =-\frac{1}{2} \partial_{M}\left(e_{b N} e^{b}\left(e_{\bar{a}}^{M} e^{\bar{a} N}\right)-e_{\bar{b} N} e^{\bar{b}}\left(e_{a}^{M} e^{a N}\right)\right) \\
& =\frac{1}{2} \partial_{M}\left(e_{b N} e^{b}\left(e_{a}^{M} e^{a N}\right)+e_{\bar{b} N} e^{\bar{b}}\left(e_{a}^{M} e^{a N}\right)\right) \\
& =\frac{1}{2} \partial_{M} \partial_{N}\left(e_{a}{ }^{M} e^{a N}\right)
\end{aligned}
$$

We next consider the terms in (A.32) depending only on the frame field. The first three terms in (A.32) yield

$$
\begin{aligned}
e_{a} \tilde{\Omega}^{a}+\frac{1}{2} \tilde{\Omega}_{a}{ }^{2}+\left.\frac{1}{2} e_{a} e_{b} \mathcal{G}^{a b}\right|_{e} & =e_{a}\left(\mathcal{G}^{a b} \partial_{N} e_{b}{ }^{N}\right)+\frac{1}{2} \mathcal{G}^{a b} \partial_{M} e_{a}{ }^{M} \partial_{N} e_{b}{ }^{N}+\frac{1}{2} e_{a} e_{b} \mathcal{G}^{a b} \\
& =\frac{1}{2} \partial_{M}\left(e_{a}{ }^{M} \mathcal{G}^{a b} \partial_{N} e_{b}{ }^{N}\right)+\frac{1}{2} e_{a} \partial_{N}\left(e_{b}{ }^{N} \mathcal{G}^{a b}\right) \\
& =\frac{1}{2} \partial_{M} e^{a M} \partial_{N} e_{a}{ }^{N}+\frac{1}{2} e^{a M} \partial_{M} \partial_{N} e_{a}{ }^{N}+\frac{1}{2} e_{a}{ }^{M} \partial_{M} \partial_{N} e^{a N} \\
& =\frac{1}{2} \partial_{M} \partial_{N}\left(e^{a M} e_{a}{ }^{N}\right)-\frac{1}{2} \partial_{M} e_{a}{ }^{N} \partial_{N} e^{a M}
\end{aligned}
$$

which, combined with the relations (A.38) and (A.39), implies

$$
\begin{aligned}
e_{a} \tilde{\Omega}^{a}+\frac{1}{2} \tilde{\Omega}_{a}^{2}+\frac{1}{2} e_{a} e_{b} \mathcal{G}^{a b}= & -\frac{1}{2}\left(\nabla^{a}\left(e_{a}{ }^{M} \bar{\nabla}^{\bar{b}} e_{\bar{b} M}\right)-\bar{\nabla}^{\bar{a}}\left(e_{\bar{a}}{ }^{M} \nabla^{b} e_{b M}\right)\right) \\
& -\left(\nabla^{a} d e_{a}{ }^{M} \bar{\nabla}^{\bar{b}} e_{\bar{b} M}-\bar{\nabla}^{\bar{a}} d e_{\bar{a}}{ }^{M} \nabla^{b} e_{b M}\right)-\frac{1}{2} \partial_{M} e_{a}{ }^{N} \partial_{N} e^{a M} .
\end{aligned}
$$

In order to evaluate the last four terms in $\mathcal{R}$ it is convenient to use the form given in (A.33). The last term in there can be written as

$$
-\frac{1}{4} \Omega_{\bar{c} a b} \Omega^{\bar{c} a b}=-\frac{1}{4}\left(f_{\bar{c} a b} f^{\bar{c} a b}+2 f_{\bar{c} a b} f^{a b \bar{c}}+2 f_{a \bar{c} b} f^{a \bar{c} b}+2 f_{\bar{c} a b} f^{b \bar{c} a}-2 f_{a \bar{c} b} f^{b \bar{c} a}\right)
$$


where we have used $f_{A b \bar{c}}=-f_{A \bar{c} b}$ which follows from the constraint (2.13). In total we find

$$
\begin{aligned}
& -\frac{1}{4}\left(f_{a b c}+f_{b c a}+f_{c a b}\right) f^{c a b}-\frac{1}{4} \Omega_{\bar{c} a b} \Omega^{\bar{c} a b} \\
& =-\frac{1}{4}\left(f_{C a b} f^{C a b}+2 f_{C a b} f^{a b C}+2 f_{a \bar{c} b} f^{a \bar{c} b}+2 f_{\bar{c} a b} f^{b \bar{c} a}-2 f_{a \bar{c} b} f^{b \bar{c} a}\right) .
\end{aligned}
$$

The first term in here is zero by the strong constraint. The results for the remaining terms are given by

$$
\begin{aligned}
f_{C a b} f^{a b C}= & -e_{a}{ }^{M} e^{a L} \partial_{N} e^{b}{ }_{M} \partial_{L} e_{b}{ }^{N}, \\
f_{a \bar{c} b} f^{a \bar{c} b}= & \frac{1}{2}\left(e_{a}{ }^{M} e^{a N} \nabla^{b} e^{\bar{c}}{ }_{M} \nabla_{b} e_{\bar{c} N}-e_{\bar{a}}{ }^{M} e^{\bar{a} N} \nabla^{\bar{b}} e^{c}{ }_{M} \nabla_{\bar{b}} e_{c N}\right), \\
f_{\bar{c} a b} f^{b \bar{c} a}-f_{a \bar{c} b} f^{b \bar{c} a}= & -\left(e_{\bar{c}}{ }^{M} e^{\bar{c} N} \nabla_{a} e_{b M} \nabla^{b} e^{a}{ }_{N}-e_{c}{ }^{M} e^{c N} \nabla_{\bar{a}} e_{\bar{b} M} \nabla^{\bar{b}} e^{\bar{a}}{ }_{N}\right) \\
& -e_{\bar{a}}{ }^{M} e^{\bar{a} L} \partial_{N} e^{b}{ }_{M} \partial_{L} e_{b}{ }^{N},
\end{aligned}
$$

which are relatively straightforward to derive by using that the covariant derivatives coincide with the ordinary derivatives by arguments similar to those used in (4.6). Inserting these into (A.43) gives together with (A.40) and (A.37) the final result displayed in (2.59).

\section{A.4 Ricci tensor in terms of $\mathcal{E}_{i j}$}

In this appendix we verify that the Ricci-type tensor $\mathcal{R}_{a \bar{b}}$ obtained from Siegel's curvature tensor coincides, upon gauge fixing, with the tensor obtained from the double field theory action (1.2) by variation with respect to $\mathcal{E}_{i j}$ as given in [11] (there denoted by $\mathcal{K}_{a b}$ ).

We start from the expression

$$
\mathcal{R}_{a \bar{b}}=R_{\bar{c} a \bar{b}}^{\bar{c}}=e_{\bar{c}} \omega_{a \bar{b}}^{\bar{c}}-e_{a} \omega_{\bar{c} \bar{b}}^{\bar{c}}+\omega_{d \bar{b}}{ }^{\bar{c}} \omega_{\bar{c} a}^{d}-\omega_{a \bar{b}}{ }^{\bar{d}} \omega_{\bar{c} \bar{d}}{ }^{\bar{c}},
$$

which after gauge fixing and upon identification of indices can be rewritten in terms of the Christoffel symbols $\Gamma$ reviewed in sec. 4.2 as

$$
R_{\bar{k} i \bar{j}}^{\bar{k}}=\mathcal{D}_{i}\left(\Gamma_{\bar{k} \bar{j}}^{\bar{k}}-\frac{1}{2} \mathcal{D}^{k} \mathcal{E}_{k j}-2 \overline{\mathcal{D}}_{j} d\right)-\overline{\mathcal{D}}_{\bar{k}} \Gamma_{i \bar{j}}^{\bar{k}}+\Gamma_{l \bar{j}}^{\bar{k}} \Gamma_{\bar{k} i}^{l}-\Gamma_{i \bar{j}}^{\bar{l}}\left(\Gamma_{\bar{k} \bar{l}}^{\bar{k}}-\frac{1}{2} \mathcal{D}^{k} \mathcal{E}_{k l}-2 \overline{\mathcal{D}}_{l} d\right) .
$$

The dilaton terms reduce to

$$
\left.R_{\bar{k} i \bar{j}}\right|_{d} ^{\bar{k}}=-2 \mathcal{D}_{i} \overline{\mathcal{D}}_{j} d+2 \Gamma_{i \bar{j}}^{\bar{l}} \overline{\mathcal{D}}_{l} d=-\left(\nabla_{i} \overline{\mathcal{D}}_{j} d+\bar{\nabla}_{j} \mathcal{D}_{i} d\right)
$$

which is consistent with the dilaton terms in $\mathcal{K}_{i \bar{j}}$ [11, where we note that in this appendix all covariant derivatives $\nabla_{i}$ are with respect to $\Gamma$.

Next, we inspect the $\mathcal{E}$-dependent terms in $\mathcal{R}_{i \bar{j}}$ :

$$
\left.R_{\bar{k} i \bar{j}}\right|_{\mathcal{E}}=\mathcal{D}_{i} \Gamma_{\bar{k} \bar{j}}^{\bar{k}}-\overline{\mathcal{D}}_{\bar{k}} \Gamma_{i \bar{j}}^{\bar{k}}+\Gamma_{l \bar{j}}^{\bar{k}} \Gamma_{\bar{k} i}^{l}-\Gamma_{i \bar{j}}^{\bar{l}} \Gamma_{\bar{k} \bar{l}}^{\bar{k}}-\frac{1}{2} \nabla_{i} \mathcal{D}^{k} \mathcal{E}_{k j}
$$

which after some computation can be rewritten as

$$
\begin{aligned}
\left.R_{\bar{k} i \bar{j}}{ }^{\bar{k}}\right|_{\mathcal{E}}= & \frac{1}{2}\left(\bar{\nabla}^{k} \overline{\mathcal{D}}_{k} \mathcal{E}_{i j}-\nabla^{k} \mathcal{D}_{i} \mathcal{E}_{k j}-\bar{\nabla}^{k} \overline{\mathcal{D}}_{j} \mathcal{E}_{i k}\right)+\frac{1}{4} g^{p q}\left(\mathcal{D}_{i} \mathcal{E}_{p j} \overline{\mathcal{D}}^{k} \mathcal{E}_{q k}+\mathcal{D}_{i} \mathcal{E}_{q k} \overline{\mathcal{D}}^{k} \mathcal{E}_{p j}\right) \\
& -\frac{1}{4}\left(\overline{\mathcal{D}}^{k} \mathcal{E}_{l j} \mathcal{D}^{l} \mathcal{E}_{i k}+\overline{\mathcal{D}}^{k} \mathcal{E}_{i j} \mathcal{D}^{l} \mathcal{E}_{l k}\right)+\frac{1}{4} g^{p q} \overline{\mathcal{D}}_{j} \mathcal{E}_{i p} \mathcal{D}^{l} \mathcal{E}_{l q}-\frac{1}{4} g^{k l} g^{p q} \overline{\mathcal{D}}_{j} \mathcal{E}_{k p} \mathcal{D}_{i} \mathcal{E}_{l q}
\end{aligned}
$$

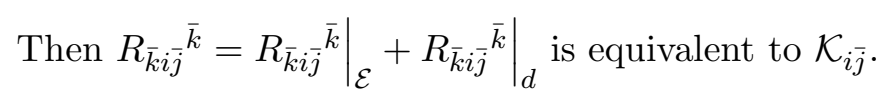




\section{References}

[1] A. Giveon, M. Porrati and E. Rabinovici, "Target space duality in string theory," Phys. Rept. 244, 77 (1994) arXiv:hep-th/9401139.

[2] M. J. Duff, "Duality Rotations In String Theory," Nucl. Phys. B 335 (1990) 610.

[3] A. A. Tseytlin, "Duality Symmetric Formulation Of String World Sheet Dynamics," Phys. Lett. B 242, 163 (1990); "Duality Symmetric Closed String Theory And Interacting Chiral Scalars," Nucl. Phys. B 350, 395 (1991).

[4] C. M. Hull, "A geometry for non-geometric string backgrounds," JHEP 0510 (2005) 065 arXiv:hep-th/0406102.

[5] C. M. Hull, "Doubled geometry and T-folds," JHEP 0707 (2007) 080 arXiv:hep-th/0605149.

[6] C. Hull and B. Zwiebach, "Double Field Theory," JHEP 0909 (2009) 099 arXiv:0904.4664 [hepth]].

[7] C. Hull and B. Zwiebach, "The gauge algebra of double field theory and Courant brackets," JHEP 0909 (2009) 090 arXiv:0908.1792 [hep-th]].

[8] O. Hohm, C. Hull and B. Zwiebach, "Background independent action for double field theory," JHEP 1007 (2010) 016 arXiv:1003.5027 [hep-th]].

[9] O. Hohm, C. Hull and B. Zwiebach, "Generalized metric formulation of double field theory," JHEP 1008 (2010) 008 arXiv:1006.4823 [hep-th]].

[10] D. S. Berman and M. J. Perry, "Generalized Geometry and M theory," arXiv:1008.1763 [hep-th].

[11] S. K. Kwak, "Invariances and Equations of Motion in Double Field Theory," JHEP 1010 (2010) 047 arXiv:1008.2746 [hep-th]].

[12] P. West, "E11, generalised space-time and IIA string theory," arXiv:1009.2624 [hep-th].

[13] D. Lust, "T-duality and closed string non-commutative (doubled) geometry," arXiv:1010.1361 [hep-th].

[14] I. Jeon, K. Lee and J. H. Park, "Differential geometry with a projection: Application to double field theory," arXiv:1011.1324 [hep-th].

[15] T. H. Buscher, "A Symmetry of the String Background Field Equations," Phys. Lett. B 194 (1987) 59, "Path Integral Derivation of Quantum Duality in Nonlinear Sigma Models," Phys. Lett. B 201 (1988) 466.

[16] W. Siegel, "Superspace duality in low-energy superstrings," Phys. Rev. D 48, 2826 (1993) arXiv:hep-th/9305073. 
[17] W. Siegel, "Two vierbein formalism for string inspired axionic gravity," Phys. Rev. D 47, 5453 (1993) arXiv:hep-th/9302036.

[18] T. Courant, "Dirac Manifolds." Trans. Amer. Math. Soc. 319: 631-661, 1990.

[19] N. Hitchin, "Generalized Calabi-Yau manifolds," Q. J. Math. 54 (2003), no. 3, 281-308, arXiv:math.DG/0209099.

[20] M. Gualtieri, "Generalized complex geometry," PhD Thesis (2004). arXiv:math/0401221v1 [math.DG]

[21] T. Kugo and B. Zwiebach, "Target space duality as a symmetry of string field theory," Prog. Theor. Phys. 87 (1992) 801 arXiv:hep-th/9201040.

[22] W. Siegel, "Fields," arXiv:hep-th/9912205. 